\title{
Stimulus manipulations permit activation of fiber subpopulations in the mouse and rat vagus
}

Yao-Chuan Chang ${ }^{1, *}$, Umair Ahmed ${ }^{1}$, Naveen Jayaprakash ${ }^{1}$, Ibrahim Mughrabi ${ }^{1}$, Qihang Lin ${ }^{2}$, Yi-Chen $\mathrm{Wu}^{1}$, Adam Abbas ${ }^{1}$, Arielle H. Gabalski ${ }^{1}$, Anna Daytz ${ }^{1}$, Jason Ashville ${ }^{1}$, Socrates Dokos $^{2}$, Tianruo Guo ${ }^{2}$, Yousef Al-Abed ${ }^{1}$, Stavros Zanos ${ }^{1, *}$

${ }^{1}$ Institute of Bioelectronic Medicine, Feinstein Institutes for Medical Research, Manhasset NY 11030, United States

${ }^{2}$ Graduate School of Biomedical Engineering, University of New South Wales, Sydney, 2052, Australia

* Corresponding authors: Yao-Chuan Chang and Stavros Zanos

\section{Abstract}

Nerve fibers of different types converge in the cervical vagus, providing sensory and motor innervation to visceral organs and mediating therapeutic and off-target effects of vagus nerve stimulation (VNS). Selectively activating fiber subpopulations, especially small, unmyelinated fibers, which constitute the majority of vagal fibers, with cervical VNS remains a challenge, hindering the study of vagal regulation of organ homeostasis and limiting the safety and efficacy of VNS therapies. We sought to select and optimize parameters that preferentially activate large, intermediate or small vagal fibers in 2 popular experimental animal species, rats and mice. VNS trains with different waveforms, pulsing frequencies and intensities were tested. Resulting engagement of fibers was quantified over 3 time scales: in the millisecond range, using stimuluselicited compound action potentials, in the second to minute range, using cardiorespiratory, vagus-mediated physiological responses and in the minute to hour range, using c-Fos expression in vagal neuronal populations in the brainstem. From those measurements, selectivity indices were compiled for different fiber types and optimal stimulus parameters were determined. Large and intermediate-size fibers are activated by trains of short-square and long-square/quasitrapezoidal pulses, respectively, at different optimal intensities in different animals. Small fibers are selectively activated by trains of $>8 \mathrm{KHz}$ frequency and relatively high stimulus intensities, that at the same time block larger fibers. Results from these manipulations were replicated in rats and mice, suggesting that they translate across species. In a computational model of vagal fibers, fiber-selectivity of KES could be explained by how fibers with the same ionic channels but different morphologies respond to $\mathrm{KHz}$ stimuli of different intensities and frequencies. This study provides a robust design and optimization framework for targeting vagal fibers of different sizes in physiological and preclinical studies of VNS in rodents. 


\section{Introduction}

The vagus nerve innervates many visceral organs and relays information between peripheral receptors, effector cells and the brain ${ }^{1}$. Cervical vagus nerve stimulation (VNS), provides access to practically all vagal fibers in a relatively minimally-invasive manner ${ }^{2}$. It has been successfully applied clinically in drug-resistant epilepsy ${ }^{3}$, depression ${ }^{4}$, and rheumatoid arthritis ${ }^{5}$, and is preclinically tested in Alzheimer's disease ${ }^{6}$, pain ${ }^{7}$, anxiety ${ }^{8}$, tinnitus ${ }^{9}$, heart failure ${ }^{10}$, diabetes ${ }^{11}$ obesity $^{12}$, and systemic ${ }^{13}$ and pulmonary hypertension ${ }^{14}$. Both therapeutic and off-target effects of cVNS, are initiated by activation of different types of vagal fibers. For example, anti-epileptic action is mostly related to large, afferent A-fibers ${ }^{15}$, cardio-inhibitory action in heart failure to intermediate-size, efferent B fibers ${ }^{16}$, and anti-inflammatory action to efferent and possibly small, afferent $\mathrm{C}$-fibers ${ }^{17,18}$. Safe and effective VNS therapies, that maximize desired and minimize off-target effects, for existing and future indications, require stimulation parameters that achieve selective activation of vagal fiber populations. Furthermore, elucidation of the role of different fibers in the many homeostatic reflexes mediated by the vagus would benefit from fiber-selective methods for engaging the afferent or efferent arms of those reflexes.

A major challenge for fiber-selective VNS, is the inverse electrical recruitment order of fibers, from large-to-small. As a result of that, off-target effects of VNS, such as the activation of large fibers innervating the larynx and pharynx, often limit its therapeutic efficacy ${ }^{19-22}$. The therapeutic potential of VNS is also hindered, considering that the small C-fibers comprise about $75 \%$ of all the fibers in cervical $\mathrm{VN}^{23}$. Several stimulation strategies have been used to alter the fiber recruitment order. Stimulus polarity, via the mechanism of anodal block, has been used to suppress activation of larger size fibers in the afferent or efferent direction ${ }^{24,25}$. However, anodal block selects mostly for fiber direction rather than fiber size, and smaller fibers are relatively insensitive to $\mathrm{it}^{25}$. Waveform manipulation, combined with stimulus polarity, has been used to differentially fibers, including slowly rising pulses ${ }^{24,26,27}$, pre-pulse ${ }^{24}$, and quasi-trapezoidal (QT) pulses $^{24,28}$. Those methods change the sensitivity of fibers in different sizes to the stimuli either through leveraging morphological differences between fibers ${ }^{29}$ or pre-condition voltage-gated ion channels ${ }^{30,31}$. Though waveform manipulation has been tested in different animal models and with varying degrees of success, testing of its effects has been limited to measuring the electrical activity of nerve fibers, without assessing the engagement of associated neuronal populations or innervated end-organs. As a consequence, the translational value of waveform manipulation in fiber-selective VNS remains unclear ${ }^{28,32}$. It is also unknown unclear whether small fibers, especially unmyelinated fibers, can be selectively targeted by manipulating stimulus waveform.

In addition to waveform manipulation, pulsing frequency has been used to selectively block nerve fibers. KHz-range electrical stimulation (KES), uses rapidly alternating rectangular or sinusoidal current to block conduction in axons, initially in somatic nerves targeting muscle force production $^{30,33}$, and more recently, in the vagus, targeting inflammation and appetite ${ }^{34,35}$. KES block effect in not limited to larger fibers; by changing the frequency and intensity, block can occur in smaller fibers, while leaving its conduction recoverable ${ }^{36}$. Part of the blocking effect of KES is thought to be related to the kinetics of axonal sodium channels ${ }^{14,37}$, even though that is under debate. It is unknown whether frequency manipulation can be used to selectively activate, rather than just block fibers, as shown in retinal study to mediate on/off cell responses ${ }^{38}$. 
In this study, we leverage stimulus waveform and pulsing frequency to achieve selective activation of vagal fibers according to their size in 2 common preclinical animal models, rat and mice. We accessed fiber engagement by VNS over 3 time scales: in the millisecond scale, by recording fiber-specific, single stimulus-evoked compound action potentials (eCAPs), in the second-to-minute scale, by registering fiber-specific physiological responses to stimulus trains, and in the minute-to-hour scale, by determining c-Fos expression in associated sensory and motor neuronal groups in the brain stem. Using those measurements, we compiled nerve fiber, physiological and neuronal selectivity indices for each fiber types and used those to optimize stimulation parameters in individual animals. We found that selective activation of large (Atype) and intermediate size (B-type) fibers is attained through waveform manipulation, using relatively low pulsing frequencies, at different optimal intensities for different subjects. Selective activation of small (C-type) fibers is attained by KES at pulsing frequencies $>8 \mathrm{KHz}$, at relatively high intensities. These results were consistent between rat and mouse subjects. Using a computational model of large, myelinated and small, unmyelinated nerve fibers to simulate how they respond to KES, we found that dynamics of sodium channels and fiber responses were both shaped by axonal size and myelin structure. Our results demonstrate that selective activation of large, intermediate or small size vagal fibers by VNS is attainable in individual subjects through an optimization procedure using waveform and frequency manipulations. Those manipulations are similar in 2 different rodent species, indicating reliance on common biophysical and ological mechanisms.

\section{Methods}

\section{Animal preparation, anesthesia, physiological instrumentation}

Forty-two adult male Sprague Dawley rats (age 2-5 months and weight between 300-550 gm) and eleven male C57BL/6 mice (2-4 months and weight between 25-30 gm) were used in the study under the approval of the Institutional Animal Care and Use Committee at The Feinstein Institutes for Medical Research. Rodents were anaesthetized using isoflurane (induction at $4 \%$ and maintenance at 1.5-2\%) and medical oxygen; anesthesia was maintained throughout the experiment. Body temperature was measured with a rectal probe and maintained between 36.5$37.5^{\circ} \mathrm{C}$ using a heating pad (78914731, Patterson Scientific) connected to a warm water recirculator (TP-700 T, Stryker). ECG was recorded by using 3-lead needle electrodes subcutaneously on the limbs and amplified using a commercial octal bio-amplifier (FE238, ADI). Breathing was monitored by using a temperature probe placed outside of the nostrils along with a bridge amplifier (FE221, ADI); the probe reported changes in air temperature during breathing movements: drop in temperature during inhalation, and rise during exhalation (Fig. $\mathrm{S} 1 \mathrm{~A}$ and $\mathrm{B}$ ). All physiological signals were first digitized and then acquired at 1-KHz (PowerLab 16/35, ADI) and visualized on LabChart v8 (all from ADInstruments Inc).

\section{Surgical preparation and vagus electrode placement}

To expose the cervical vagus nerve $(\mathrm{cVN})$ in the rat model, a midline $3 \mathrm{~cm}$ skin incision was given on the neck (Fig. S1A). Salivary glands were separated, and muscles were retracted to reach the carotid bundle. Under a dissecting microscope, the right $\mathrm{cVN}$ was isolated first at the caudal end of nerve and then at rostral end of nerve. The middle portion, between the two isolated sites was left intact within carotid bundle to minimize the extent of surgical 
manipulation and trauma to the nerve. After isolation of the nerve, a pair of custom-made, tripolar cuff electrodes was placed on the caudal and rostral sites relative to omohyoid muscle (Fig. S1A). The cuff electrodes were made using a polyimide substrate and sputter-deposited iridium oxide contacts for low electrode impedances and stable stimulation characteristics ${ }^{39-41}$. Electrode contacts had dimensions of $1418 \times 167 \mu \mathrm{m} 2$ with an edge-to-edge spacing of $728 \mu \mathrm{m}$ and center-to-center spacing of $895 \mu \mathrm{m}$. Typical individual electrode impedances in saline ranged from 0.5 to $1.5 \mathrm{k} \Omega$. The distance between the stimulating electrode (center contact of tripolar cuff) to the most proximal recording electrode on the nerve was measured roughly 5 to 6 $\mathrm{mm}$. Silicone elastomer (Kwiksil by World Precision Instruments) was placed around the cuff to minimize current leakage during stimulation. In the mouse model, all surgical procedures were identical except the left cVN was targeted. In addition, for direct laryngeal muscle measurement, the thyroid cartilage was exposed by separating the sternohyoid muscle at the midline using blunt dissection. Using a $29 \mathrm{G}$ insulin syringe, a shallow slit was made in the thyroid cartilage just lateral and inferior to the laryngeal prominence. With the needle bevel facing up, the two PTFEcoated platinum-iridium wires were carefully inserted into the underlying laryngeal muscles through the slit guided by the syringe needle.

\section{Vagus nerve recording and stimulation}

Neural activity from each contact on the recording electrode was amplified, digitized (30KS/s, 16bit resolution) and filtered (60-Hz notch), using a 32-channel RHS2000 stim/record headstage and 128ch Stimulation/Recording controller (Intan Technologies); recordings were single-ended, relative to a ground lead placed in the salivary gland. Nerve stimulation was delivered in constant current mode as trains of pulses using an STG4008 stimulus generator (Multichannel Systems). For all experiment related to waveform manipulation, the stimulation protocols were composed of monophasic pulse with varying pulse width, intensity, polarity, and waveform shape. Monophasic pulses were used here to yield lower threshold and simpler stimulus artifact shape. Even though pulses were not charge-balanced, it is unlikely that during these acute experiments with low pulsing frequency, significant charge build-up occurred. In particular, fully randomized single pulse with $30-\mathrm{s}$ on and $10-\mathrm{s}$ off at $1 \mathrm{~Hz}$ were used to access the neural response, whereas stimulus trains of 10 -s durations with identical type of pulse at $30 \mathrm{~Hz}$ were randomly delivered to evoked discernable physiological response. For experiment related to frequency manipulation, all the stimuli were delivered in biphasic form except for probing pulse, to maintain the charge balancing across the neural interface and minimize the neural injury. All the stimuli were constructed as train form with consistent 10-s duration but with varying frequency, pulse width, and intensity, and randomly delivered from a range. The stimulation configuration was tripolar (cathode-center or cathode-corner) as it outperforms in terms of protection of current leakage for all experiments. There were at least 15-s long pauses between successive trains to ensure that physiological measurements had reached a steady state before a new train was delivered.

In all experiments with neural recording, we initially determined the "neural threshold" (NT) as the lowest stimulus intensity for a $100-\mu$ s duration pulses that evoked a discernable evoked potential at the recording electrode. The physiological threshold (PT), which evoked visible (5$10 \%$ ) heart rate/respiratory change (usually 3 or $4 \times \mathrm{NT}$ ), was used in experiment when no neural signals were recorded and for all KES experiments. The details of each experiment can be found in Table S1 and S2. Specifically, to access the neural activity in response to the KES with one stimulation cuff, we designed the waveform, which is combined with KES with low frequency, 
$1 \mathrm{~Hz}$ probing pulse, as the low frequency probing pulse does not contribute significantly to physiological effect ${ }^{42}$. For each probing pulse, a $30-\mathrm{ms}$ window $(5 \mathrm{~ms}$ before, $25 \mathrm{~ms}$ after the onset) is opened in the 10-s KES train, to improve the signal-to-noise ratio for further evoked neural signal processing in next section.

\section{Identification and analysis of neural and EMG signals}

Raw nerve signal traces from both electrodes were filtered using a $1 \mathrm{~Hz}$ high-pass filter to remove the DC component. Stimulus-evoked compound action potentials (eCAPs) elicited from individual pulses or from trains of pulses, were extracted, by averaging individual sweeps of nerve recording traces around the onset of pulses (waveform manipulation experiments) or probing pulse (frequency manipulation experiments). A custom-made buffer amplifier was used to record the induced voltage on the electrode during stimulation. Stimulation artifact was suppressed offline for waveform manipulation experiment by a recently proposed method which subtracts the trace of the stimulation electrode voltage from the eCAPs with proper template matching and an edge effect removal algorithm ${ }^{43}$. For frequency manipulation, due to the saturation of artifact voltage buffer, same artifact removal algorithm has not been applied.

Given the rough estimation of distance between the recording and stimulation electrodes (5-6 $\mathrm{mm}$ ), we fine tune the distance in analysis so that the latency windows can align well with the A, B- and C-fiber prominent peaks with pre-defined conduction velocity ranges for each fiber type (A: $5-120 \mathrm{~m} / \mathrm{s} ; \mathrm{B}: 2-8 \mathrm{~m} / \mathrm{s} ; \mathrm{C}: 0.1-0.8 \mathrm{~m} / \mathrm{s})^{44}$. Fig. S1C shows representative eCAP, including activity of different fiber types and EMG. Signals from both contacts in the recording electrode, proximal and distal to the stimulating electrode, were collected (solid blue and dashed red traces in Fig. S1C). This allowed to distinguish between neural and EMG signal components. For the given electrode spacing A- and B-fibers had short latencies ( $<3 \mathrm{~ms}$, red and green windows), while slower $\mathrm{C}$-fibers occurred at longer latencies $(>6 \mathrm{~ms} \text {, yellow window })^{43}$. To discriminate $\mathrm{C}$ fiber components from EMG, we reasoned that $\mathrm{C}$-fiber volleys should show a latency difference between the proximal and distal recording contact, spaced apart by a distance of $895 \mu \mathrm{m}$, of 1-2 ms, whereas EMG signals should occur simultaneously on both recording contacts ${ }^{43}$ (Fig. S1C, grey window), with time window around 2-6 ms (identified with neuromuscular junction blocking agent in our previous study ${ }^{42}$ ).

\section{Analysis of physiological signals}

We computed the magnitude of EMG response from respective eCAPs as the peak-to-trough amplitude of the (typically biphasic) response within the EMG window (Fig. S1C, grey window); that amplitude was then normalized by the maximum EMG amplitude in that subject. Using a custom algorithm, ECG peaks corresponding to the R waves were identified, and heart rate (HR) was computed from R-R intervals. We defined stimulus-induced change in $H R(\Delta H R)$ as the difference between the mean HR during a 10-s epoch before the onset of the stimulus train ("Before-VNS") and the mean HR during the stimulus train ("During-VNS"), divided the mean pre-stimulus HR. In recordings from the nasal temperature sensor, we identified peaks (end of expiration) and troughs (end of inspiration). We defined the interval between two successive peaks (or two successive troughs) as breathing interval (BI). We defined the stimulus-elicited change in breathing interval $(\Delta \mathrm{BI})$ as the difference between the mean pre-stimulus and the mean during-stimulus BI. For those cases without breath during stimulation period, the breathing 
interval between last breath of pre-stimulus and first breath post-stimulus was used as mean during-stimulus BI. The measured signals and corresponding derived variables (ECG and $\triangle \mathrm{HR}$, and nasal sensor temperature and $\triangle \mathrm{BI}$ ) are shown in Fig. S1B. All the analyses were performed using MATLAB 2016a software (MathWorks, Natik, MA, USA).

\section{Immunohistochemistry}

Rats delivered VNS intermittently for 30 minutes (10 seconds on, 50 seconds off), were deeply anesthetized with isoflurane and transcardially perfused using $250 \mathrm{ml}$ of $0.9 \%$ saline after $1.5 \mathrm{hrs}$. The brains were removed immediately and post-fixed for 2 days in $4 \%$ paraformaldehyde in 0.1 M PBS. After post fixation, the brainstem was precut by razor blade and sectioned in vibratome

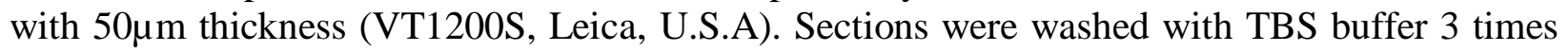
for 5 minutes and subjected to antigen retrieval using 1X SignalStain ${ }^{\circledR}$ Citrate Unmasking Solution (Cell signaling). The citrate buffer was bought to boiling temperature and added to the sections. The well plate was incubated at $85^{\circ} \mathrm{C}$ for 10 minutes. The plate was allowed to cool down and the sections were washed with TBST buffer (1X TBS buffer $+0.025 \%$ tween 20 ). $5 \%$ normal donkey serum and $0.3 \%$ Triton-X100 in TBS buffer was used as blocking buffer and the sections were blocked for 1 hour at room temp. Sections were first stained with c-Fos and then Choline Acetyltransferase (ChAT) was performed subsequently. C-Fos staining was the done by incubating the sections with the c-Fos antibody (Abcam, ab190289) at 1:1000 dilution in blocking buffer for 3 days placed in shaking incubator at $4^{\circ} \mathrm{C}$. Section were rinsed with the TBST buffer $3 \times 5$ times and incubated in the donkey anti-rabbit secondary antibody labeled with alexa-fluro 488 (1:500) in blocking buffer for 2 hours at room temperature. Sections were washed $3 \times 5$ minutes in TBST buffer and incubated with ChAT antibody (Sigma, AB144) at 1:100 dilutions overnight at $4^{\circ} \mathrm{C}$ in the blocking buffer. Sections were rinsed the next day with $3 \times 5$ minutes in TBS buffer and incubated with anti-goat secondary antibody labelled with alexa fluro 555 (1:200) for 2 hours in room temperature. Sections were rinsed in TBST buffer 3 times and incubated with DAPI 1:1000 dilution in TBS buffer for 1 hour at room temperature. The section was then rinsed two times with TBS buffer and mounted on to Poly-L-lysine coated glass slides. Cover glass was secured on the top of the sections with VECTASHIELD® PLUS Antifade Mounting Medium (Vector labs, H-1900). Sections from naïve, sham and different VNS treatment group were processed in parallel.

c-Fos and ChAT expression in the nucleus tractus solitarius (NTS) and dorsal motor nucleus of the vagus nerve (DMV) was captured using all-in-one fluorescence microscope under 20X objective (BZ-X800, Keyence, U.S.A.). After processed by commercial imaging stitching software (BZ-X800 Analyzer), the orientation of the stitched images was first adjusted for crossanimal comparison and consistency. To correct non-uniform illumination and attenuate background, the original image was subtracted from background approximation image estimated from morphological opening method. Cells expressing c-Fos and ChAT were counted bilaterally in 3-4 sections/brain using customized cell count tool, with sections taken across $-13.68 \mathrm{~mm} \pm$ $250 \mu \mathrm{m}$ according to Breagma, with ROIs for NTS and DMV regions were marked by anatomical expert. Counting was performed bilaterally and represented by counts per regions by an investigator blinded to the treatment conditions. All the post-processing techniques were performed using MATLAB 2016a. 
CAP, physiological, and neural selective indices

To evaluate the fiber selective performance of tested stimulation parameters, for different types of fibers, we defined CAP selectivity indices (CSIs) which aim to maximize target and minimize off-target fiber activity. The CSIs for A-, B-, C-fibers are:

$$
\begin{aligned}
& \operatorname{CSI}(A-\text { fiber })=\frac{A}{B+C+\varepsilon} \\
& \operatorname{CSI}(B-\text { fiber })=\frac{{ }_{B}}{A+C+\varepsilon} \\
& \operatorname{CSI}(C-\text { fiber })=\frac{C}{A+B+\varepsilon}
\end{aligned}
$$

where $\mathrm{A}, \mathrm{B}, \mathrm{C}$ are normalized evoked fiber activity within each animal, and a small constant $\varepsilon$ is used to prevent the overflow due to the extremely small fiber activity in the denominator.

Similar to CSIs, based on an existing relationship/models between fiber activity and physiological response: A-fiber to evoked EMG, B-fiber to HR, and C-fiber to BI (Fig. S2) ${ }^{42}$, we defined physiological selective indices (PSIs) which aim to maximize desired and minimized non-desired physiological effects corresponding to different type of fiber activation. The PSI for A-, B-, C-fibers defined as:

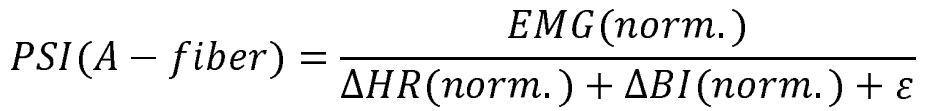

$$
\begin{aligned}
& P S I(B-\text { fiber })=\frac{\Delta H R(\text { norm } .)}{E M G(\text { norm. })+\Delta B I(\text { norm } .)+\varepsilon} \\
& P S I(C-\text { fiber })=\frac{\Delta B I(\text { norm })}{E M G(\text { norm })+\Delta H R(\text { norm })+\varepsilon}
\end{aligned}
$$

where $\mathrm{EMG}($ norm.), $\Delta \mathrm{HR}$ (norm.), and $\Delta \mathrm{BI}$ (norm.) are normalized physiological responses within each animal, and a small constant $\varepsilon$ is used to prevent the overflow, same as CSI. To quantify the performance of PSIs using different stimulation parameters across animals, a 1st order Gaussian model was computed to capture the relationship between computed PSIs and different stimulation intensities.

Finally, to quantify the immunohistochemistry results in brainstem regions, we further defined neural selective index (NSI) for sensory neurons (mostly related to C-fibers), which is:

$$
N S I \text { (sensory) }=\left|\frac{\Delta c F \text { os in NTS }(\%)}{\Delta c F \text { os in DMV }(\%)}\right|
$$

where $\Delta \mathrm{c}$-Fos in NTS and DMV are normalized with respect to the average number of expressed neurons in corresponding regions in sham group animals.

\section{Computational model of vagal fibers and KES}

All simulations were implemented in COMSOL Multiphysics v. 5.4 (COMSOL Inc., Burlington, MA). Our nerve fiber model structure and parameters were adapted from the McIntyre, Richardson and Grill (MRG) ${ }^{45}$ and the Schwarz, Reid and Bostock (SRB) models ${ }^{46}$. Two major nerve fiber subtypes were simulated; myelinated A fiber and unmyelinated $\mathrm{C}$ fibers. Ion channels are modelled on the nodes of Ranvier, based on the formulations of the SRB model according to

$$
i_{\text {ion }}=i_{N a}+i_{K f}+i_{K s}+i_{L}
$$

As shown in Figure S7A, the extracellular environment was modelled by a $1000-\mu \mathrm{m}$ long, 40$\mu \mathrm{m}$ diameter cylinder surrounding the $1 \mathrm{D}$ nerve fiber ${ }^{47}$. Two $50-\mu \mathrm{m}$ electrodes (50 $\mu \mathrm{m}$ apart) 
were placed on the surface of the cylinder with the electrode edges forming a $60^{\circ}$ angle with the nerve fiber. The first electrode was the cathode and the second was designated as ground.

The stimulus waveform included a wide range of frequencies ranging from $0.1-\mathrm{KHz}$ to $12-\mathrm{KHz}$ sinusoid KES, with a duration of $50 \mathrm{~ms}$. A no-flux (i.e insulating) boundary condition was implemented for $\mathrm{Vi}$ and $\mathrm{Ve}$ at the ends of the fiber. The mesh for the myelinated fibers was set to a total of 20 elements for each myelin segment and a size of $0.5 \mu \mathrm{m}$ for each node segment. The mesh for nonmyelinated fibers was set to a total of 20 elements for each fiber segment, defined as being the same length as the myelin segments of the myelinated fibers. The length of the nodes was set to $1 \mu \mathrm{m}$ in all myelinated fibers ${ }^{45}$. The length of the myelin compartment was also modelled as a function of the myelin diameter ${ }^{45,48}$. The node and myelin diameters used in the model were estimated based on histological data from rat cervical nerves ${ }^{48}$. The model's predictive ability was validated by in vivo compound nerve action potential recordings from the same animals ${ }^{48}$.

Node and myelin structures in the model fibers were characterized by different partial differential equations (PDEs). Myelin was approximated by a distributed resistance in parallel with a capacitance (Fig. S7B). We approximated the MRG double cable structure by a singlecable model of the vagus nerve to reduce the computational complexity. The membrane dynamics at the node follows SRB formulations. Models for all fiber types shared ion channel parameters but had fiber-specific physical parameters. All model parameters are listed in Table S3.

The extracellular potential distribution Ve was calculated using:

$$
\nabla\left(-\frac{1}{\rho_{e}} \nabla\left(V_{e}\right)\right)=0
$$

where $\rho_{e}$ is the extracellular resistivity. The intracellular potential Vi was calculated separately for the myelin and node compartments:

$$
\begin{gathered}
-\nabla\left(\frac{r_{n}}{\rho_{n}}\left(\nabla V_{i}\right)\right)+2 C_{n} \frac{\partial V_{i}}{\partial t}=-2\left(i_{i o n}-C_{n} \frac{\partial V_{e}}{\partial t}\right) \\
-\nabla\left(\frac{r_{m y}}{\rho_{m y}}\left(\nabla V_{i}\right)\right)+2 C_{m y} \frac{\partial V_{i}}{\partial t}=2 C_{m y} \frac{\partial V_{e}}{\partial t}
\end{gathered}
$$

where $\mathrm{rn}$ and rmy are the nodal and myelin radius respectively. Membrane potential Vm was determined from the difference between the intracellular and extracellular potentials.

\section{Statistics}

Analysis of Covariance (ANCOVA) was used to compare the neural responses (A-, B-, C-), physiological responses (EMG, HR, BI), and proposed CSIs and PSIs for different stimulus manipulations (categorical independent variable) and intensity (continuous independent variable). Linear regression was used to compare the same stimulus parameter with different intensity. One-way analysis of variance (ANOVA) and Tuckey's post-hoc tests were used to compare the histological results in brainstem, and two sample t-test was used for corresponding NSI. Comparison were deemed statistically significant for $\mathrm{p}<0.05$ for all analyses. All statistical analyses were performed on MATLAB (Mathworks). 


\section{Results}

We used 2 main stimulus manipulations, in addition to stimulus intensity, to target activation of $\mathrm{A}, \mathrm{B}$ and $\mathrm{C}$ vagal fiber types: waveform manipulation and frequency manipulation. For waveform manipulation, we considered 3 different waveforms, at different stimulus intensities: short square pulses (SP, $100 \mu$ s pulse width), long square pulses (LP, $600 \mu$ s pulse width) and quasi-trapezoidal pulses (QT, consisting of a $100 \mu \mathrm{s}-$ long plateau and a $2500 \mu \mathrm{s}-\mathrm{long}$ exponentially-decaying falling phase). The performance of the 3 waveforms with respect to fiber selectivity was evaluated by recording eCAP responses to random sequences of single stimuli of the 3 waveforms, to compile CAP selectivity indices (CSIs) for each of 3 fiber types (A, B and $\mathrm{C}$ ), and by collecting fiber-specific physiological responses to trains of stimuli, to compile corresponding physiological selectivity indices (PSIs). With waveform manipulation, we were able to selectively engage A-fibers (SP stimuli), or B-fibers with minimal or no engagement of A- or C-fibers (LP and QT stimuli). For frequency manipulation, we delivered trains of square pulse stimuli at different frequencies in the $\mathrm{KHz}$ range, at multiple intensities and compared them to intensity-matched stimulus trains with $30 \mathrm{~Hz}$ pulsing frequency, further identify the cut off value of frequency that elicit distinct physiological response patterns other than low frequency range. The performance of $\mathrm{KHz}$ pulsing frequency with respect to fiber selectivity was evaluated by compiling PSIs, using physiological responses to stimulus trains, by compiling CSIs using eCAP responses to test pulses probing fiber excitability, and by histologically assessing activation of neural populations in the brainstem associated with different fiber types. Using frequency manipulation, we were able to selectively engage $\mathrm{C}$-fibers at frequencies above $5-\mathrm{KHz}$, with reduced engagement of A- or B-fibers, and the results were further validated by cFos expression in brainstem associated with VN and neural selectivity index (NSI) for sensory region. Both waveform and frequency manipulations were tested in 2 rodent species, rats and mice, with similar results. Finally, a possible role for sodium ion channels, resulting from the morphological differences between fibers, in the C-fiber selectivity of KHz-range frequency stimuli was explored in a computational model of unmyelinated vagal fibers.

\section{Waveform manipulation allows selective activation of A- and B-fibers}

It has been demonstrated before ${ }^{24,25}$ that single-pulse stimuli elicit eCAPs that depend on stimulus waveform, intensity and polarity configuration (Fig. 1). In cathode-corner polarity, the fiber recruitment order is usually A-, followed by B-fibers, with no evidence of C-fiber engagement; fiber amplitude is monotonically increasing with stimulus intensity for all 3 waveforms, SP, LP and QT (Fig. 1A, panels a-c). In the cathode-center polarity, SP stimuli produce the same fiber recruitment order, though the response is greater than with cathodecorner stimuli of the same stimulus intensity (Fig. 1A-d). With the same polarity, LP and QT stimuli of increasing intensity produce progressively smaller A-fiber responses, whereas B- and C-fiber responses, once elicited, become progressively larger (Fig. 1A-e and 1A-f, respectively). Intensity at intermediate levels for both LP and QT stimuli are associated with greater activation 
of B-fibers compared to that of A- or C-fibers (Fig. 1A panels, panels e-f). The statistics of normalized amplitude associated with fiber responses demonstrate the same trend (Fig. 1B). To quantify the degree of A-, B- and C-fiber selectivity of each of the 3 waveforms, we compiled fiber type-specific CAP selectivity indices (CSIs), with the targeted fiber in the numerator and the non-targeted fibers in the denominator. For A-fibers, maximum CSI is achieved at threshold intensity with all 3 waveforms, but is highest with SP stimuli (Fig. 1C). For B-fibers, SP has the worst performance, with a moderate increase at higher intensities, LP and QT waveforms have maximal CSIs at intermediate intensity levels and QT outperforms the other 2 in terms of maximum CSI and average CSI across all intensities (Fig. 1D). Intensities of 3-6×NT for LP and 4-7 $\times$ NT for QT stimuli are associated with greater activation of B-fibers compared to that of Aor C-fibers.

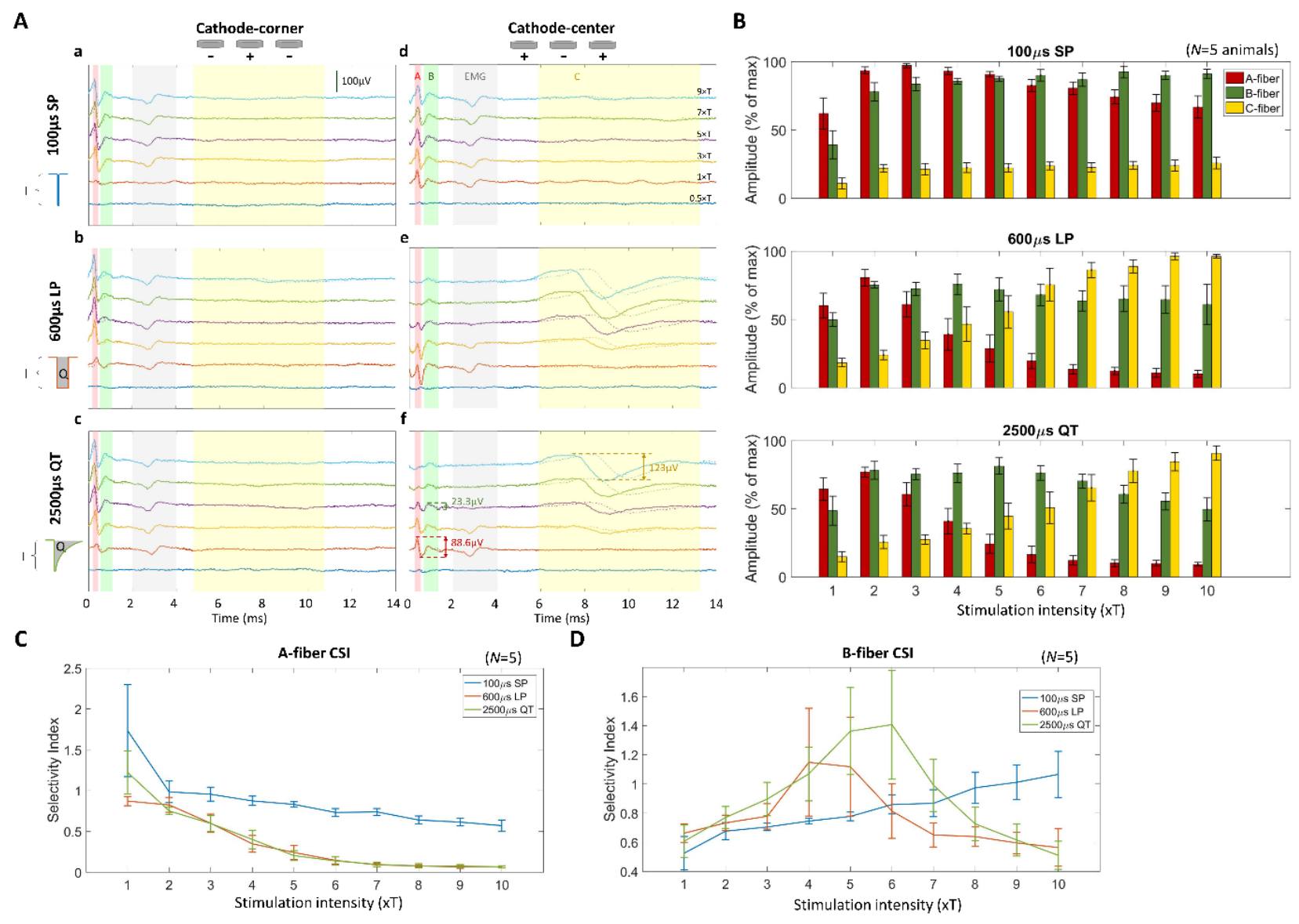

Figure 1: Stimulus-evoked compound action potentials (eCAPs), fiber activation amplitudes and resulting CAP selectivity indices (CSIs) in response to A- and B-fiber-selective stimulus waveforms, in the rat model. (A) Representative eCAPs evoked by different stimulus waveforms: $100 \mu$ s square pulse (short square pulse, SP; panel a, d), $600 \mu$ s square pulse (long square pulse, LP; panel b, e), and 100 $\mu \mathrm{s}-$ plateau followed by a $2500 \mu \mathrm{s}$ exponentially decaying pulse (quasi-trapezoidal pulse, QT: panel c, f). Shown are representative eCAP examples in response to cathode-center and cathode-corner polarities and a range of stimulus intensities, from 1 to 10 times thresholds $(\times N T)$ in a single animal. Traces from both proximal (solid) and distal (dash) contacts of the recording electrode are shown and each trace represents the average of triggers. A-, B-, and C-fiber activation and stimulus-evoked EMG activity, are calculated as peak-to-trough amplitude of eCAP components with different latencies and their overlap with latency 
windows corresponding to the conduction velocities of different fibers (A-fiber: red; B-fiber: green; Cfiber: yellow; EMG: grey). (B) Mean ( \pm SE, $N=5$ animals) normalized fiber activity evoked by the 3 stimulus waveforms of cathode-center polarity, at different stimulus intensities, in 5 animals. (ANCOVA, $p<0.05$ for waveform, across all fibers (A-, B-, C-), $p<0.05$ for intensity except of B-fibers $(0.21)$ ). (C) Mean ( \pm SE, $N=5$ animals) CSI values for A-fibers, for each of the 3 stimulus waveforms, as a function of stimulus intensity. (ANCOVA, $p<0.05$ for waveform and intensity, $p>0.05$ for interaction). (D) Same as $(\mathrm{C})$ but for B fibers. (ANCOVA, $p=0.27$ and $p=0.75$ for waveform and intensity respectively, $p<0.05$ for interaction).

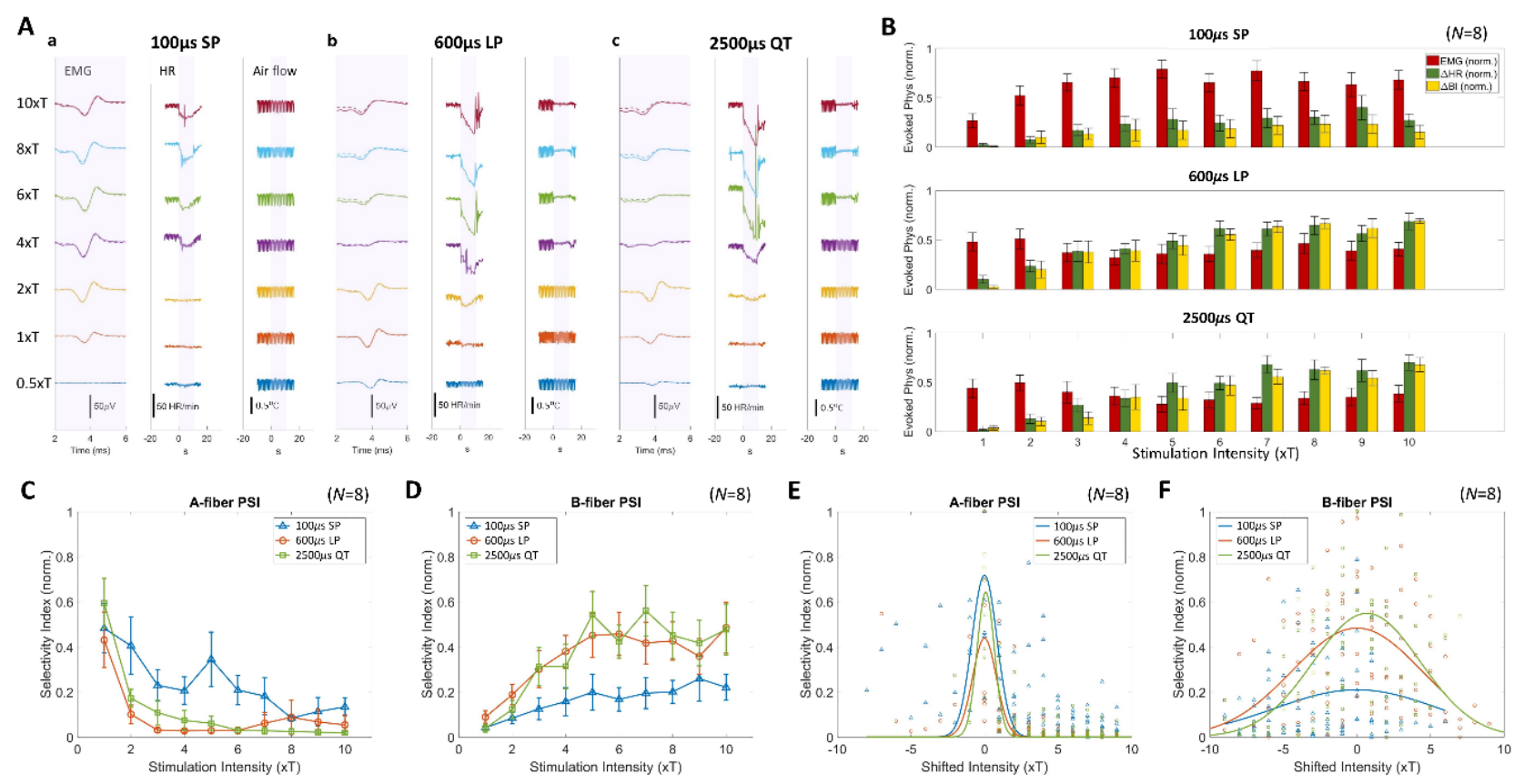

Figure 2: VNS-elicited changes in physiological parameters, magnitude of physiological effects and resulting physiological selective indices (PSIs) in response to A- and B-fiber selective stimulus waveforms, in the rat model. (A) Representative laryngeal EMG (left for each panel), heart rate (HR, middle of each panel), and breathing responses (air flow, right of each panel) elicited by stimulus trains of 3 different waveforms, short pulse (SP), long pulse (LP) and quasi-trapezoidal (QT), for a range of stimulus intensities ( 0.5 to $10 \times \mathrm{NT})$. (B) Mean ( $\pm \mathrm{SE}, \mathrm{N}=8$ animals) normalized EMG (red bars), HR $(\triangle \mathrm{HR}$, green bars) and breathing interval responses $(\triangle \mathrm{BI}$, yellow bars) elicited by the 3 stimulus waveforms, for different stimulus intensities. (ANCOVA, $p<0.05$ for waveform, across all physiological responses (EMG, HR, BI), $p<0.05$ for intensity, across all physiological responses except for EMG (0.9)). (C) Mean ( \pm SE, $N=8$ animals) normalized A-fiber PSI values associated with each of the stimulus waveforms (SP: blue, LP: red, QT: green trace), as a function of stimulus intensity. (ANCOVA, $p<0.05$ for waveform and intensity, $p>0.05$ for interaction). (D) Same as (C) but for B-fibers. (ANCOVA, $p<0.05$ for waveform and intensity, $\mathrm{p}<0.05$ for interaction). (E) Normalized A-fiber PSI values for each of the 3 waveforms plotted against stimulus intensity, re-aligned to the value associated with the maximum PSO for each animal and waveform, so the intensity shift of 0 corresponds the intensity producing the maximum PSI for each animal. Also shown are 1-D Gaussian function fits to the data for each of the 3 waveforms. Rmse for each fits: 0.235(SP), 0.126(LP), 0.111(QT). (ANCOVA, $p<0.05$ for waveform and absolute intensity shift, $p>0.05$ for interaction). (F) Same as (E) but for B-fiber PSI values. Rmse for each fits: 0.188(SP), 0.240(LP), 0.254(QT). (ANCOVA, $p<0.05$ for waveform and absolute intensity shift, $p<0.05$ for interaction). 


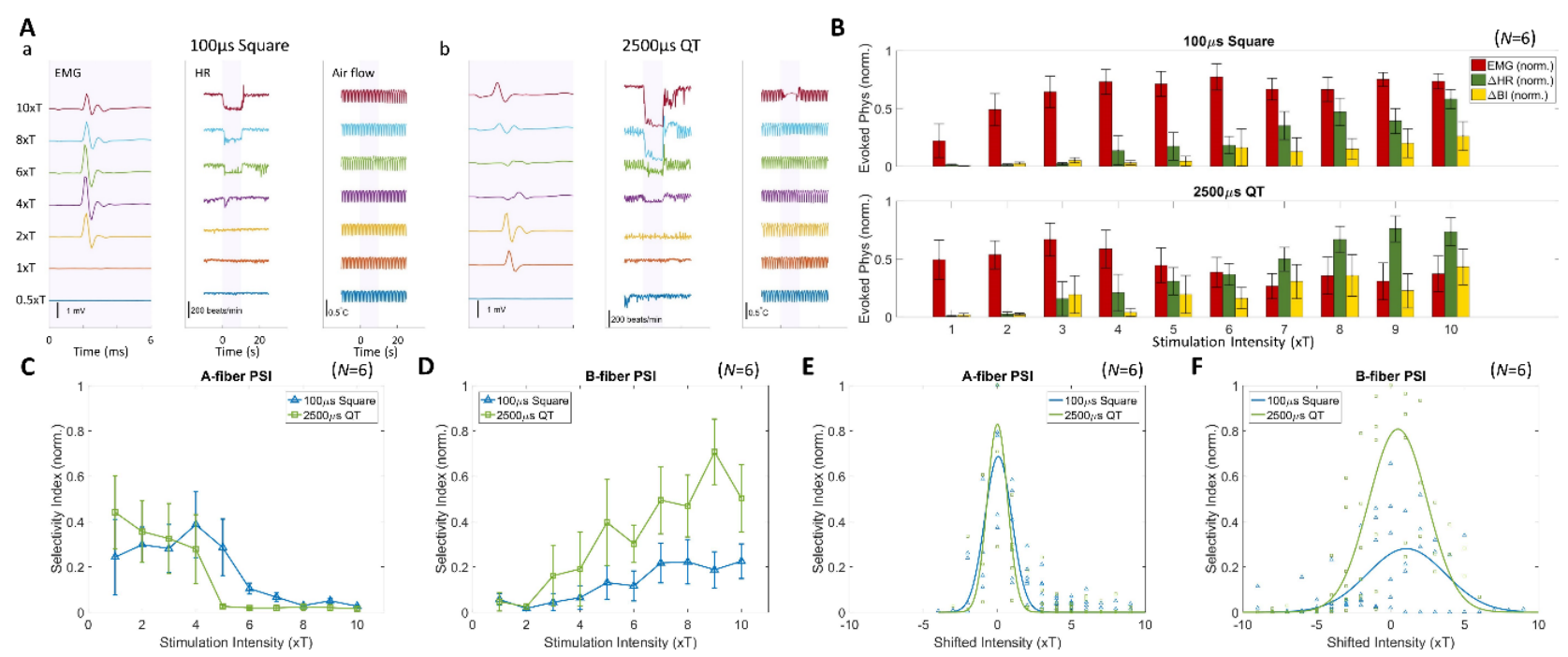

Figure 3: VNS-elicited changes in physiological parameters, magnitude of physiological effects and resulting physiological selective indices (PSIs) in response to A- and B-fiber selective stimulus waveforms, in the mouse model. (A) Representative laryngeal EMG (left for each panel), heart rate (HR, middle of each panel), and breathing responses (air flow, right of each panel) elicited by stimulus trains of 2 different waveforms, short pulse (SP: panel a) and quasi-trapezoidal (QT: panel b), for a range of stimulus intensities $(0.5$ to $10 \times \mathrm{PT}(\mathrm{EMG})$, close to NT). (B) Mean $( \pm \mathrm{SE}, \mathrm{N}=6$ animals $)$ normalized EMG (red bars), HR ( $\triangle \mathrm{HR}$, green bars) and breathing interval responses ( $\triangle \mathrm{BI}$, yellow bars) elicited by the 2 stimulus waveforms, for different stimulus intensities. (ANCOVA, $p<0.05$ for waveform, across all physiological responses except for BI, $p<0.05$ for intensity, across all physiological responses except for EMG (0.73)). (C) Mean ( \pm SE, N=6 animals) normalized A-fiber PSI values associated with each of the stimulus waveforms (SP: blue, LP: red, QT: green trace), as a function of stimulus intensity. (ANCOVA, $p=0.55$ for waveform and $p<0.05$ intensity, $p>0.05$ for interaction). (D) Same as (C) but for B-fibers. (ANCOVA, $p<0.05$ for waveform and intensity, $p<0.05$ for interaction). (E) Normalized A-fiber PSI values for each of the 2 waveforms plotted against stimulus intensity, re-aligned to the value associated with the maximum PSO for each animal and waveform, so the intensity shift of 0 corresponds the intensity producing the maximum PSI for each animal. Also shown are 1-D Gaussian function fits to the data for each of the 3 waveforms. Rmse for each fits: 0.134(SP), 0.128(QT). (ANCOVA, $p=0.86$ for waveform and $p<0.05$ for absolute intensity shift, $\mathrm{p}>0.05$ for interaction). (F) Same as (E) but for B-fiber PSI values. Rmse for each fits: 0.140(SP), 0.224(QT). (ANCOVA, $p<0.05$ for waveform and absolute intensity shift, $p<0.05$ for interaction).

When delivered as stimulus trains ( $10 \mathrm{~s}$ duration, $30 \mathrm{~Hz}$ ) the 3 waveforms produce, distinct types of physiological responses resulting from activation of different fibers: EMG results from Afiber activation, changes in heart rate $(\Delta \mathrm{HR})$ from B-fibers and changes in breathing interval $(\triangle \mathrm{BI})$ from C-fibers (Fig. S2) ${ }^{42}$. With SP stimuli, increasing intensity results in increasing, and eventually saturating EMG, with modest $\triangle \mathrm{HR}$ and $\triangle \mathrm{BI}$ responses at higher intensities (Fig. 2Aa, Fig. $3 \mathrm{~A}-\mathrm{a} /$ mouse). With LP and QT stimuli, EMG is suppressed, all the while robust $\Delta \mathrm{HR}$ and $\triangle \mathrm{BI}$ responses are elicited (Fig. 2A-b and -c, Fig. 3A-b/mouse). Overall, trains with SP stimuli produce the largest EMG responses across all stimulus intensities, while trains with LP and QT stimuli produce larger $\triangle \mathrm{HR}$ and $\triangle \mathrm{BI}$ responses, especially at intermediate and higher intensities, with significantly smaller EMG effects (Fig. 2B, Fig. 3B/mouse). When the 3 types of physiological responses are combined into fiber-specific physiological selectivity indices (PSIs), SP stimuli are on average more selective for A-fibers (Fig. 2C, Fig. 3C/mouse), and LP and QT stimuli are more selective for B-fibers (Fig. 2D, Fig. 3D/mouse). The intensity levels associated 
with peak PSI values for A-fibers are typically in the range 1-3×PT, and for B-fiber in a wider range, varying across animals. When PSI curves from each animal are aligned at the "optimal" intensity, PSI falls with intensity around the peak value (Fig.2E/3E and 2F/3F, for A- and Bfibers in rat/mouse respectively). At optimal intensity, QT outperforms the other 2 waveforms in terms of its B-fiber selectivity (One-way ANOVA, $p<0.05$, Fig. S3, Fig. S4/mouse), consistent with CSI findings in rat.

\section{Frequency manipulation allows selective activation of C-fibers}

KHz-range electrical stimulation (KES), has been used to block conduction in vagal fibers according to their diameter, including small C-fibers ${ }^{36}$. We sought to assess whether KES can be used to activate vagal C-fibers, while minimally activating larger, A- and B-fibers. Physiological responses to KES trains of different frequencies were compared to those elicited by $30 \mathrm{~Hz}$ trains of the same duration, intensity and PW (Fig. 4). $\triangle \mathrm{HR}$ and $\triangle \mathrm{BI}$ effects of 1-KHz trains are similar in magnitude to those of $30 \mathrm{~Hz}$ trains (Fig. 4A-c), indicating similar levels of activation of Band C-fibers, respectively. At 5-Khz and $12.5-\mathrm{KHz}$, high intensity stimulation results in similar $\triangle \mathrm{BI}$ responses as $30-\mathrm{Hz}$ trains $(p>0.05)$ but with minimal $\Delta \mathrm{HR}$ responses $(p<0.05)$, indicating engagement of C-fibers without engaging B-fibers (Fig. 4A-a, b). On average, across a wide range of intensities, the higher the frequency, the smaller the $\Delta \mathrm{HR}$ effect similar $\Delta \mathrm{BI}$ effects (Fig. 4B). To determine the frequency and intensity cut-off values beyond which selective Cfiber activation occurs, trains of stimuli with identical pulse width $(40 \mu \mathrm{s})$ were delivered at different KHz-range frequencies and intensities. At frequencies of $5-\mathrm{KHz}$ or above, $\Delta \mathrm{HR}$ responses across intensities were minimal (Fig. 4C), whereas significant $\triangle \mathrm{BI}$ responses were registered at high intensities, $8-10 \times \mathrm{PT}$ (Fig. 4D). In experiments in mice, KES of increasing intensity elicited similar $\triangle \mathrm{BI}$ effects as $30 \mathrm{~Hz}$ stimulation, with large intensity $(15-25 \times \mathrm{PT})$, but with a much smaller $\Delta \mathrm{HR}$ response and stronger suppression at low intensity (1-3×PT) (Fig. 6Ab). 
A

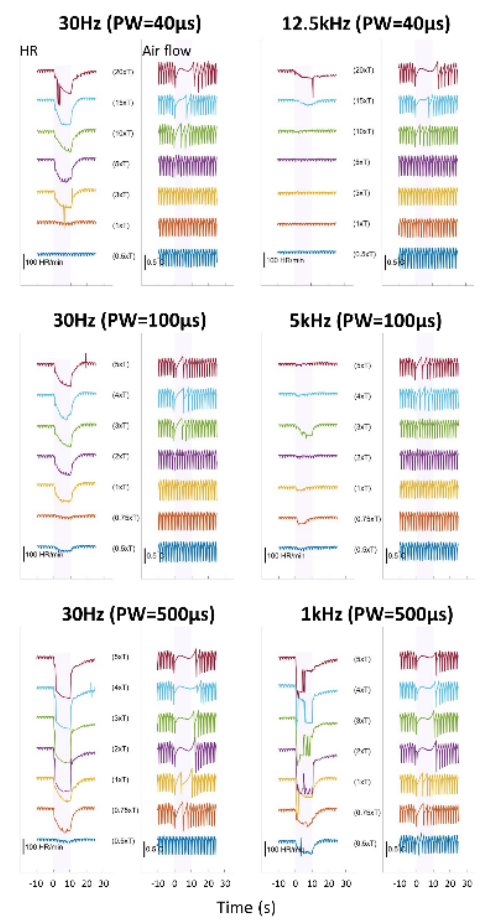

B
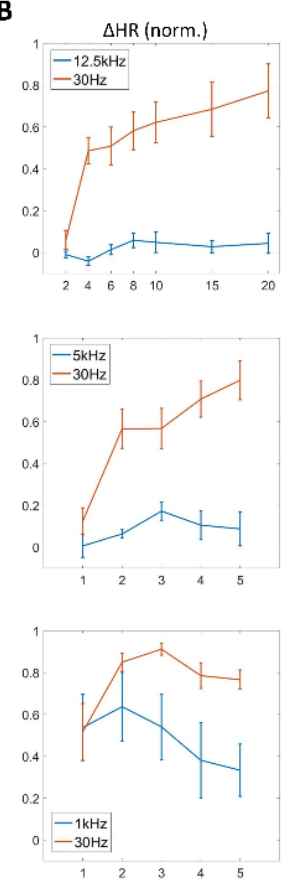

Simulation Intensity (XPT)

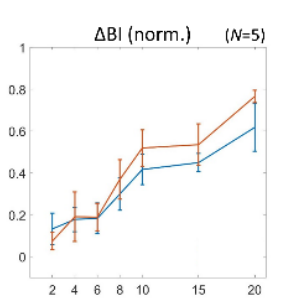

C

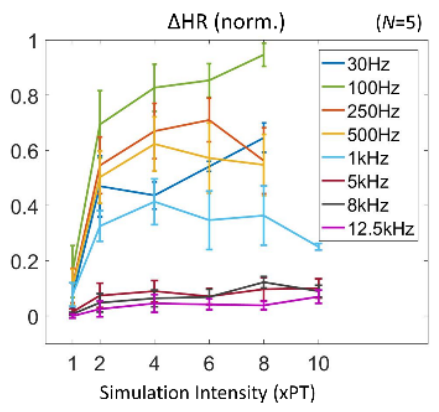

D
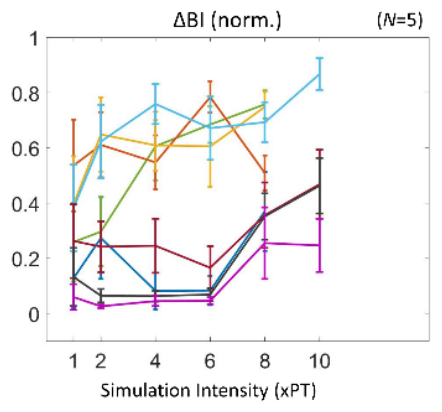

Figure 4: Physiological responses to VNS trains of different KHz-frequencies, compared to $30 \mathrm{~Hz}$ trains of stimuli with comparable pulse widths (PW), in the rat model. (A) Representative heart rate (HR) and breathing responses (airflow) elicited by stimuli of 3 different $\mathrm{KHz}$-frequencies: $1 \mathrm{KHz}(500 \mu \mathrm{s}$ PW), $5 \mathrm{KHz}(100 \mu \mathrm{s} \mathrm{PW})$ and $12.5 \mathrm{KHz}(40 \mu \mathrm{s} \mathrm{PW})$, next to their $30 \mathrm{HZ}$, PW- and intensity-matched controls from the same animal (panels a, b and c, respectively). All stimulus trains were 10-s-long, as marked by the light blue-shaded areas. (B) Mean ( \pm SE, $N=5$ animals) normalized $\triangle \mathrm{HR}$ and $\Delta \mathrm{BI}$ responses for each of the 3 frequency stimuli $(1 \mathrm{KHz}, 5 \mathrm{KHz}, 12 \mathrm{KHz}$; blue curves in panels a, b and c, respectively) along with responses to their corresponding $30 \mathrm{~Hz}$ controls (red curves in each panel), as a function of stimulus intensity. (HR: ANCOVA, $p<0.05$ for all frequency pairs and intensity except for $1 \mathrm{KHz}$ ( $p=0.73$ for intensity), and $p<0.05$ for interaction. BR: ANCOVA, $p>0.05$ for all frequency pairs ( 0.49 for $12.5 \mathrm{KHz}, 0.051$ for $5 \mathrm{KHz}, 0.99$ for $1 \mathrm{KHz}$ ) and $p<0.05$ for intensity, and $p>0.05$ for interaction). (C) Mean ( $\pm \mathrm{SE}, \mathrm{N}=5$ animals) normalized $\triangle \mathrm{HR}$ responses to trains of stimuli of varying frequencies (ranging from $30 \mathrm{~Hz}$ to $12.5 \mathrm{~Hz}$, shown in different color curves) and of identical $\mathrm{PW}(40 \mu \mathrm{s})$, as a function of stimulus intensity. (ANCOVA, $p<0.05$ for frequency and intensity, and $p<0.05$ for interaction). (D) Same as $(\mathrm{C})$ but for normalized $\triangle \mathrm{BI}$ responses. (ANCOVA, $p<0.05$ for frequency and intensity, and $p<0.05$ for interaction).

To document fiber excitability changes that could explain the fiber-specific physiological responses to KES, single "probing" pulses were delivered at regular intervals (1-s) throughout KES trains, at 8-KHz, and resulting eCAPs were compiled. Short-width probing pulses $(100 \mu \mathrm{s})$ elicit A- and B-fiber responses that abruptly disappear at KES intensities above $3 \times \mathrm{PT}$ and $7 \times \mathrm{PT}$, respectively (Fig. 5A). Long-width probing pulses (600 $\mu$ s) elicit C-fiber responses, which are progressively suppressed as KES intensity increases (Fig. 5B). Overall, both A-fibers and EMG responses are suppressed at intensities between 1-3×PT (red bars in Fig. 5C, D). B-fibers are progressively suppressed between $1-8 \times \mathrm{PT}$, which correlates with the minor moderate $\Delta \mathrm{HR}$ responses seen at those intensities (green bars in Fig. 5C, D); notably, $\Delta \mathrm{HR}$ responses are sustained but minor at intensities $>8 \times \mathrm{PT}$ (Fig. 5D). C-fibers are also suppressed progressively as intensity increases, but at a slower rate than A- or B-fibers (yellow bars in Fig. 5C); at the same 
time, $\Delta \mathrm{BI}$ responses gradually increase (yellow bars in Fig. 5D). This slower suppression rate, along with the gradual engagement of more $\mathrm{C}$-fibers by stimuli of increasing intensity, creates an "intensity window" (7-20×PT) in which A- and B-fibers are maximally suppressed, and C-fibers are partially suppressed but still excitable. Similar findings are found in mice, though optimal intensities for C-fiber activation are generally higher (10-30×PT, Fig. 6B); of note, threshold intensities in mice were much smaller than in the rat $(11.6 \mu \mathrm{A}$ vs $221.4 \mu \mathrm{A})$. This is further demonstrated by CSIs and PSIs for each fiber type: for A- and B-fibers, they both decrease with increasing KES intensity (Fig. 5E-a, b, respectively), whereas for C-fibers, both indices are maximal at KES intensities $>7 \times \mathrm{PT}$ (Fig. 5D-c, Fig. 5E). In mice, we found more varying intensities associated with $\triangle \mathrm{HR}$ response suppression and $\Delta \mathrm{BI}$ responses across animals, thus leading to more distinct optimal window for C-fiber, but the trend remained similar (Fig. 6C, D). It is worth noting that we calculated PSIs with EMG set to a constant (0.5), as we were not able to obtain laryngeal EMG under continuous KES condition due to electrical interference. To test whether addition of probing pulses to KES trains altered the patterns of physiological responses, in some rats, we compared the physiological responses, $\triangle \mathrm{HR}$ and $\triangle \mathrm{BI}$, to KES alone (without probing pulses) with the responses to KES with 100 or $600 \mu$ s-long probing pulses. No significant differences were found, across a range of KES intensities (Fig. S5).

\section{A}

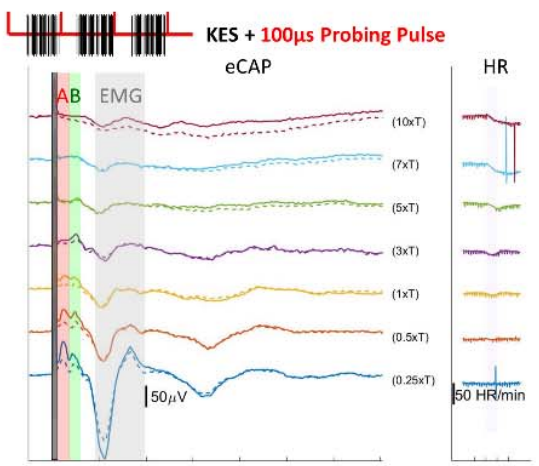

B
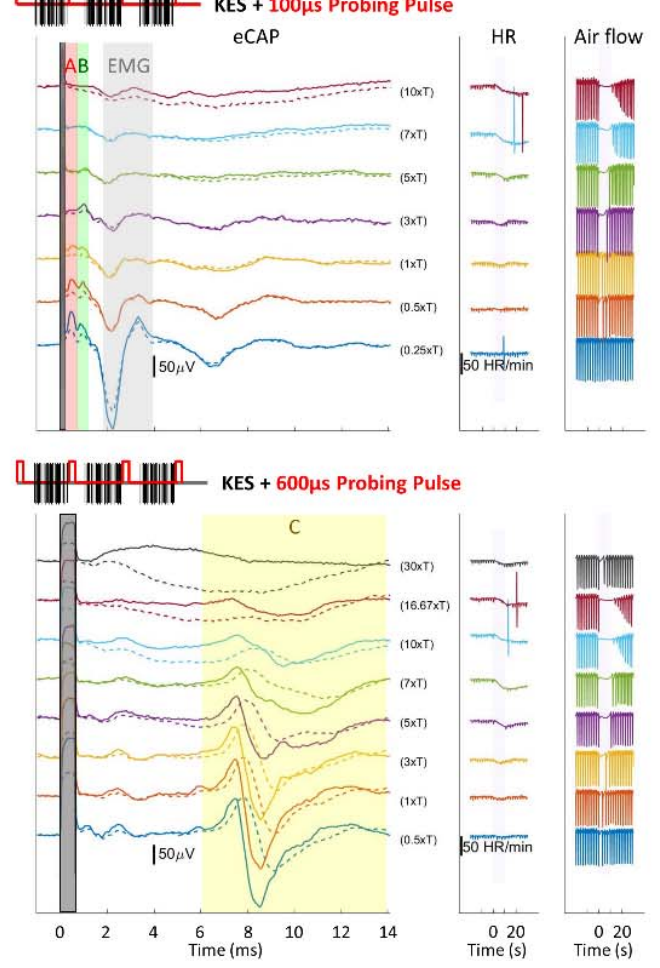

C
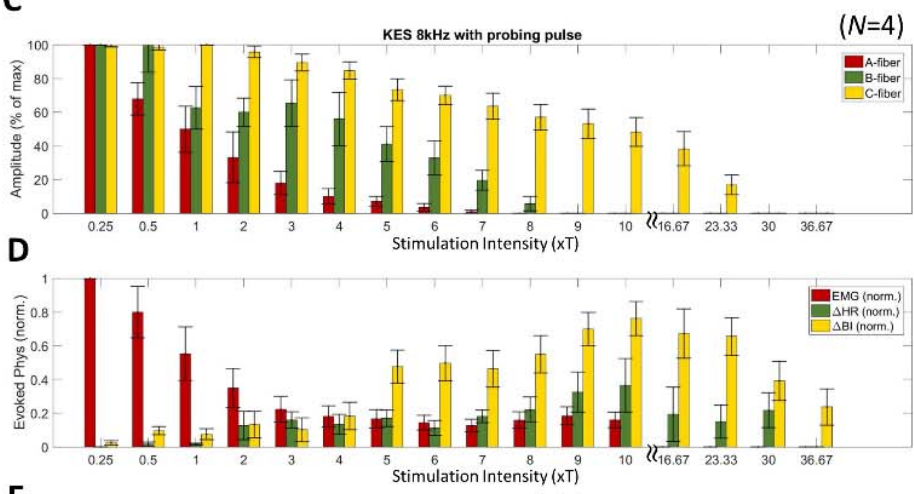

E
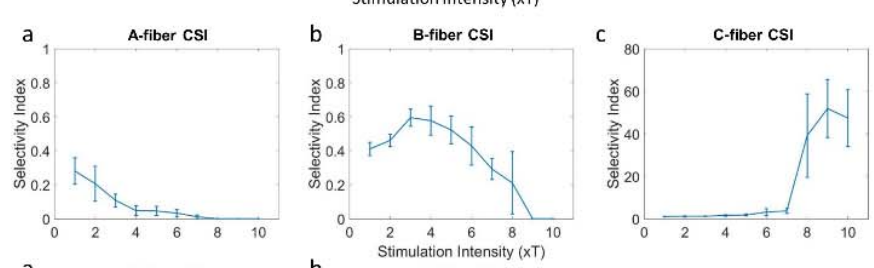

$\mathbf{F}$
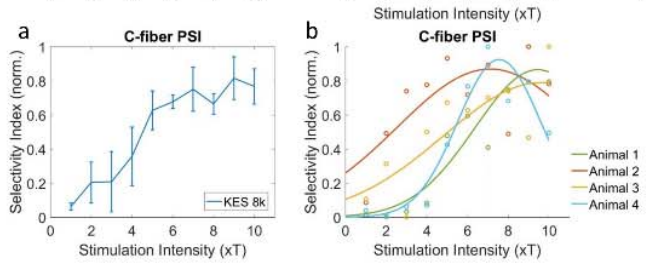

Figure 5: eCAPs, elicited physiological effects and resulting CAP and physiological selectivity indices (CSI and PSI, respectively), in response to 8-KHz frequency electrical stimulation of the vagus (KES) of different intensities, in the rat model. (A) Representative eCAPs, $\triangle H R$, and $\triangle B I$ responses by KES in a single animal, at different stimulus intensities ( 0.25 to $10 \times \mathrm{PT})$. eCAPs were compiled by delivering, concurrently with KES, single, monophasic, $100 \mu \mathrm{s}-$ long "test" pulses every $1 \mathrm{~s}$, 10 times in total, to evoke A-fiber and laryngeal EMG activity. Stimulus intensities are expressed in units 
of threshold intensity, defined as the PT of KES (0.5 to 10×PT). (B) Same as (A), but with $600 \mu$ s-long test pulses delivered concurrently with KES, to evoke C-fiber activity. (C) Mean ( \pm SE, $N=4$ animals) of normalized amplitude of A-, B-, C-fiber activity (red, green and yellow vars, respectively) evoked by test pulses (100 $\mu \mathrm{s}$ : A- and B-fiber, $600 \mu \mathrm{s}$ : C-fiber), during KES of different intensities. (Linear Regression, $p<0.05$ for intensity, across all fiber responses (A-, B-, C-)). (D) Mean ( $\pm \mathrm{SE}, \mathrm{N}=4$ animals) of normalized magnitude of physiological responses (EMG, red; $\triangle \mathrm{HR}$, green; $\triangle \mathrm{BI}$, yellow), elicited by KES of different intensities, in 4 animals. (Linear Regression, $p<0.05$ for intensity, across all physiological responses (EMG, HR, BI)). (E) Mean (+/-SE) values of CSI for A- (a), B- (b) and C-fibers (c) as function of KES intensity, in 4 animals. (ANCOVA, $p<0.05$ for intensity and all types of CSI). (F) Normalized PSIs for C-fibers as function of KES intensity (a) Mean (+/-SE) values of normalized PSIs. (Linear regression, $p<0.05$ for intensity) (b) Normalized PSI values for C-fibers cross animals, and Gaussian function is fitted to show the distribution of PSIs evoked by KES stimulus. Average Rmse for the fits: 0.169 .




Figure 6: Elicited physiological effects and resulting physiological selectivity indices (PSI), in response to 8-KHz frequency electrical stimulation of the vagus (KES) of different intensities, in the mouse model. (A) Representative $\triangle \mathrm{HR}, \triangle \mathrm{BI}$, and diaphragm EMG (associated with breathing pattern) responses by $30 \mathrm{~Hz}$ and KES in a single animal, at different stimulus intensities. Stimulus intensities are expressed in units of threshold intensity, defined as the PT of KES ( 1 to $25 \times \mathrm{PT})$. (B) Mean $( \pm \mathrm{SE}, \mathrm{N}=5$ animals) of normalized magnitude of physiological responses ( $\triangle \mathrm{HR}$, green; $\triangle \mathrm{BI}$, yellow), elicited by KES of different intensities. (Linear regression, HR: $p=0.156$, BR: $p=0.32$, for intensity). (C) Mean (+/-SE) values of PSI for C-fibers as function of KES intensity, in 5 animals. (Linear Regression, $p<0.05$ for intensity). (D) Normalized PSI values for C-fibers cross animals, and Gaussian function is fitted to show the distribution of PSIs evoked by KES stimulus. Average Rmse for the fits: 0.211.

\section{Stimulus manipulations permit selective activation of vagal neurons in the brainstem}

Even though waveform and frequency manipulations allow preferential activation of different fiber types in the vagus, it is unclear whether such manipulations can also affect the activity level of sensory and motor vagal neurons associated with those fibers. To determine that, we delivered

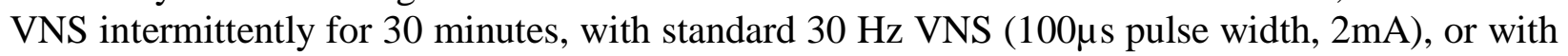
$8-\mathrm{KHz}$ VNS $(40 \mu \mathrm{s}, 2 \mathrm{~mA}, 8-10 \times \mathrm{PT})$, and measured c-Fos ${ }^{+}$immunoreactivity of single neurons in the ipsilateral and contralateral (to VNS) brainstem. In particular, we looked in the nucleus tractus solitarius (NTS), a sensory region receiving afferent vagal information form $A \beta-, A \gamma-$, $\mathrm{A} \delta$ - and mostly $\mathrm{C}$-fibers ${ }^{49,50}$, and in the dorsal motor nucleus of the vagus (DMV), a motor region with $\mathrm{ChAT}^{+}$cells providing efferent cholinergic, mostly $\mathrm{A} \alpha$ - and $\mathrm{B}$-fiber fibers, to the vagus (Fig. 7A). In the ipsilateral NTS, the $30 \mathrm{~Hz}$ VNS group has $516.3 \pm 32.16{\mathrm{c}-\mathrm{Fos}^{+}}^{+}$cells ( $314 \%$ greater than the sham group), whereas the KES group has $358.9 \pm 24.83{\mathrm{c}-\mathrm{Fos}^{+}}^{+}$cells (188\% greater than the sham). In the ipsilateral DMV, $30 \mathrm{~Hz}$ VNS resulted in $19.08 \pm 1.98 \mathrm{c}-F o s^{+}$ cells $(900 \%$ greater than sham), whereas KES in $8.17 \pm 1.52$ cells (328\% greater than naïve) (Fig. 7C). Interestingly, $30 \mathrm{~Hz}$ VNS also resulted in a small increase of c-Fos ${ }^{+}$cells in contralateral NTS and DMV compared to the sham group $(297 \pm 44.19$ and $4.917 \pm 0.95,131 \%$ and $170 \%$ compared with sham), whereas KES did not have a significant contralateral effect (Fig. 7B (left), and Fig. $7 \mathrm{C}(\mathrm{left}))$. Cell counts in the sham stimulation group were not different than naïve, in neither of the 2 brain regions, ipsilateral or contralateral (Fig. 7B and 7C). Overall, $30 \mathrm{~Hz}$ VNS resulted in increased cell activation in ipsilateral NTS and even more in ipsilateral DMV, indicating a more "efferent selective" effect, whereas KES resulted in a comparable increase cell activation in NTS with a much smaller activation in DMV, indicating a more "afferent-selective" effect (Fig. 7D). The same trend was shown using neural selectivity indices (NSIs) for sensory region in the ipsilateral side (Fig. 7E). It worth to note that the KES trigger relatively unilateral vagal activation, potentially through blocking the most of A- and B-fibers. 

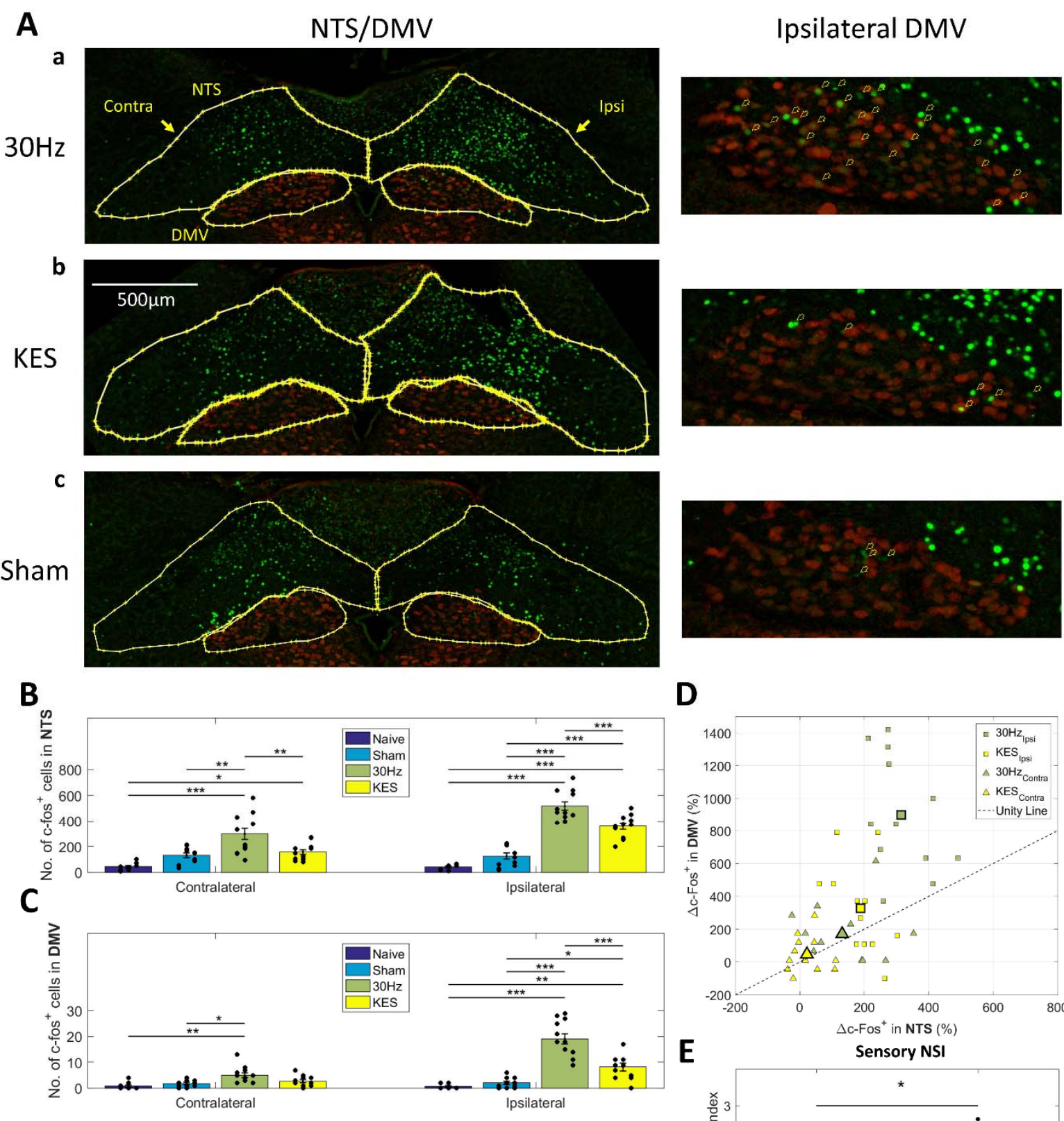

D

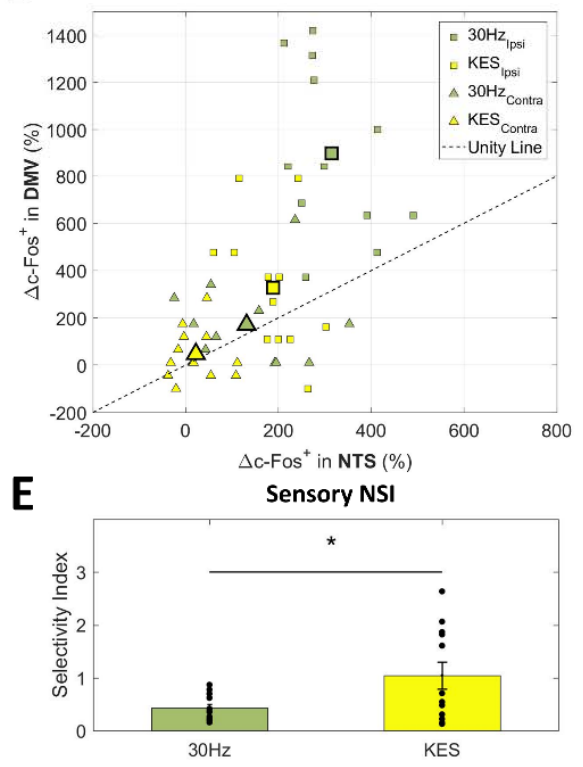

Figure 7: c-Fos expression in sensory and motor vagal brainstem regions after $30 \mathrm{~Hz}$ and $8 \mathrm{KHz}$ VNS, in the rat model. (A) Representative immunohistochemistry images of sections across ipsilateral and contralateral, to VNS, sensory and motor brainstem regions (yellow contours): nucleus tractus solitaries (NTS) and dorsal motor nucleus of the vagus (DMV) (anterior-posterior: $-13.68 \mathrm{~mm} \pm 250 \mu \mathrm{m}$ relative to Breagma, medial-lateral: $\pm 2 \mathrm{~mm}$ relative to mid sagittal plane, dorsal-ventral: $7.2-8.2 \mathrm{~mm}$ relative to brain vertex), each stained for c-Fos (green) and ChAT (red). Inset show ipsilateral DMV at higher magnification; arrows point to cells positive for c-Fos. (a) After $30 \mathrm{~min}$ of intermittent $30 \mathrm{~Hz} \mathrm{VNS}$ (10-s ON and 50-ss OFF, 30 cycles). (b) After 30 min of intermittent 8 KHZ VNS (KES), of the same cyclic settings. (c) After sham stimulation (electrode placed on nerve, no stimulation delivers). (B) $\mathrm{c}-\mathrm{Fos}^{+}$ 
cell numbers (mean \pm SE) in contralateral and ipsilateral NTS, in different group of animals: naïve (dark blue), sham stimulation (light blue), $30 \mathrm{~Hz}$ VNS (light green), KES (yellow). Each group consisted of 3 animals and each animal contributed 3-4 sections from which cell numbers were calculated. Statistical comparisons between groups used one-way ANOVA and Tuckey's post-hoc tests $(* p<0.05, * * p<0.005$, $* * * p<0.0005)$. (C) Same as (B), but for DMV. (D) Normalized c-Fos ${ }^{+}$cell numbers (\% difference in cFos+ cell numbers with respect to average cell numbers in sham group) counted in DMV vs. those counted in NTS (ipsilateral: square dots, contralateral: triangle dots), from all sections from $30 \mathrm{~Hz} \mathrm{VNS}$ group of animals (green dots) and from the KES group of animals (yellow dots). Mean values shown with black outlined dots. (E) Computed NSI (mean \pm SE), derived from change of c-Fos ${ }^{+}$expression with respect to the average of sham groups, for ipsilateral NTS and DMV regions from all sections from $30 \mathrm{~Hz}$ VNS group of animals (green) and from the KES group of animals (yellow). Statistical comparisons between groups used two-sample t-test $\left(*_{p}<0.05\right)$.

\section{Morphological properties of fibers may be responsible for differential responses to KES}

Given the known contributions of the size and shape of neurons in determining their response properties to stimuli in other neural systems systems ${ }^{51-53}$, we studied whether the different morphological properties of myelinated and unmyelinated fibers could be, at least in part, responsible for the fiber-selective effects of KES documented in this study. In particular, we hypothesized that the diameter of single axons and the presence of myelin around them affect sodium channel activation or blocking under KES. We simulated voltage responses to KES of a large, myelinated fiber, representative of efferent A and B-fibers (myelin diameter: 5- $\mu \mathrm{m}$, axonal diameter: 2.6- $\mu \mathrm{m}$ ) and of a small, unmyelinated fiber, representative of afferent C-fibers (axonal diameter: $1.3-\mu \mathrm{m}$ ), placed at the same radial distance from the stimulating electrode (Fig. S7). The physical parameters of the two fibers were estimated based on histological data from rat cervical vagus (see Fig. S8)]. Our simulations show that the response of the two fiber types to KES is distinct across a range of frequencies and intensities (Fig. 8A). At frequencies below 1$\mathrm{KHz}$, both fiber types are monotonically activated with increasing intensity, with no signs of block (Fig. 8A-a, b, c). At around 2-KHz, large myelinated fiber is activated at low intensities (2.5-5 $\mu \mathrm{A})$ and blocked at higher intensities (4-6 $\mu \mathrm{A})$. However, small unmyelinated fiber is activated at intensities above $6 \mu \mathrm{A}$. That means the "activation windows" of the two fiber types demonstrate partial overlap at this frequency (Fig. 8A-d). Those activation windows move further apart at higher frequencies, $4-\mathrm{KHz}$ and $8-\mathrm{KHz}$ (Fig. 8A-e, f), ultimately stabilizing at 12$\mathrm{KHz}$ (Fig. 8A-g). At KES frequencies $>=8-\mathrm{KHz}$, large fiber is strongly activated at low intensities (Fig. 8A-f, Fig. 8B-a1), and blocked at intermediate and high intensities, in which the membrane voltage begins to passively follow the stimulus current and remain depolarized without any elicited action potentials (APs) (Fig. 8B-a2, a3). At the same time, small unmyelinated fiber remains inactive at low and intermediate intensities (Fig. 8B-a4, a5) and is recruited at higher intensities, with robust generation of APs (Fig. 8B-a6). The KES-induced differential activation of myelinated and unmyelinated fibers predicted by our simulations agrees with the in vivo electrophysiological data (Fig. 4C and 5D). . Our simulations show that the response of the two fiber types to KES is distinct across a range of frequencies and intensities (Fig. 8A). At frequencies below $1-\mathrm{KHz}$, both fiber types are monotonically activated with increasing intensity, with no signs of block (Fig. 8A-a, b, c). At around 2-KHz, large myelinated fiber is activated at low intensities (2.5-5 $\mu \mathrm{A})$ and blocked at higher intensities (4-6 $\mu \mathrm{A})$. However, small unmyelinated fiber is activated at intensities above $6 \mu \mathrm{A}$. That means the "activation windows" of the two fiber types demonstrate partial overlap at this frequency (Fig. 
8A-d). Those activation windows move further apart at higher frequencies, $4-\mathrm{KHz}$ and $8-\mathrm{KHz}$ (Fig. 8A-e, f), ultimately stabilizing at 12-KHz (Fig. 8A-g). At KES frequencies >=8-KHz, large fiber is strongly activated at low intensities (Fig. 8A-f, Fig. 8B-a1), and blocked at intermediate and high intensities, in which the membrane voltage begins to passively follow the stimulus current and remain depolarized without any elicited action potentials (APs) (Fig. 8B-a2, a3). At the same time, small unmyelinated fiber remains inactive at low and intermediate intensities (Fig. 8B-a4, a5) and is recruited at higher intensities, with robust generation of APs (Fig. 8B-a6). The KES-induced differential activation of myelinated and unmyelinated fibers predicted by our simulations agrees with the in vivo electrophysiological data (Fig. 4C and 5D).

Fiber selectivity of KES is also reflected in the differential sodium channel dynamics in the 2 fiber types, under identical KES parameters. When myelinated fibers are blocked (Fig. 8B-a2, a3), sodium channel activation $\left(\mathrm{m}^{3}\right)$ and inactivation $(\mathrm{h})$ gating variables become entrained with the extracellular voltage, which is driven by KES (Fig. 8B-b2, b3). Under the same stimulation conditions, the gating variables $\mathrm{m}^{3}$ and $\mathrm{h}$ in unmyelinated fibers remain stable (Fig. 8B-b4, b5), and demonstrate normal physiological opening and closing with high stimulus intensities (Fig. 8B-b6). To isolate the contribution of myelin and axonal size, we simulated 8-KHz-induced spiking activity in an un-myelinated fiber with an identical axonal diameter as myelinated large fibers $(2.6 \mu \mathrm{m})$. Excitation and block thresholds of a 2.6- $\mu \mathrm{m}$ un-myelinated fiber is lower than a 1.3- $\mu \mathrm{m}$ un-myelinated fiber (Fig. 8C). The presence of myelin (e.g. in a 2.6- $\mu$ m myelinated fiber) further reduces the excitation and block thresholds (Fig. 8C). 

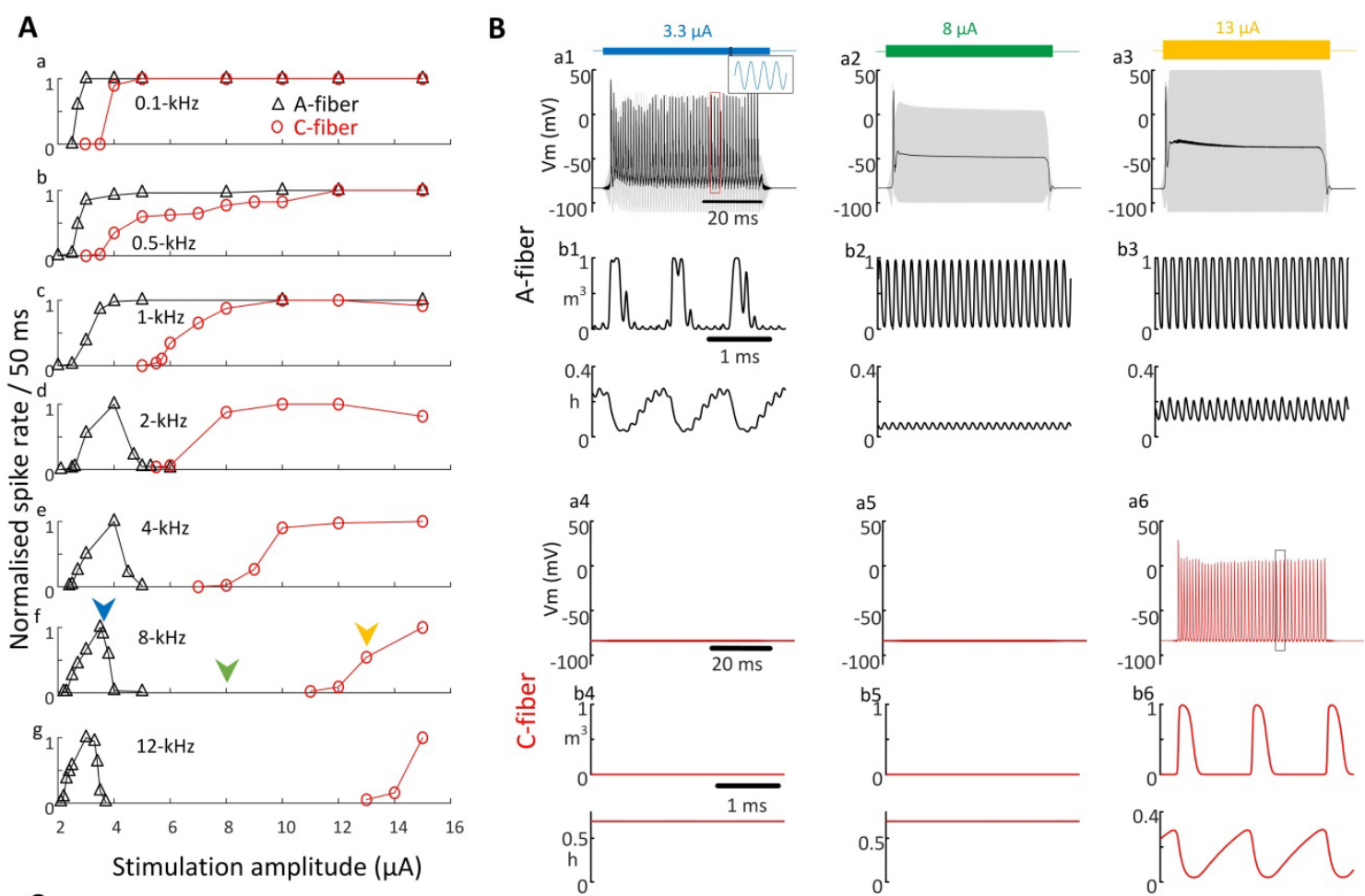

C

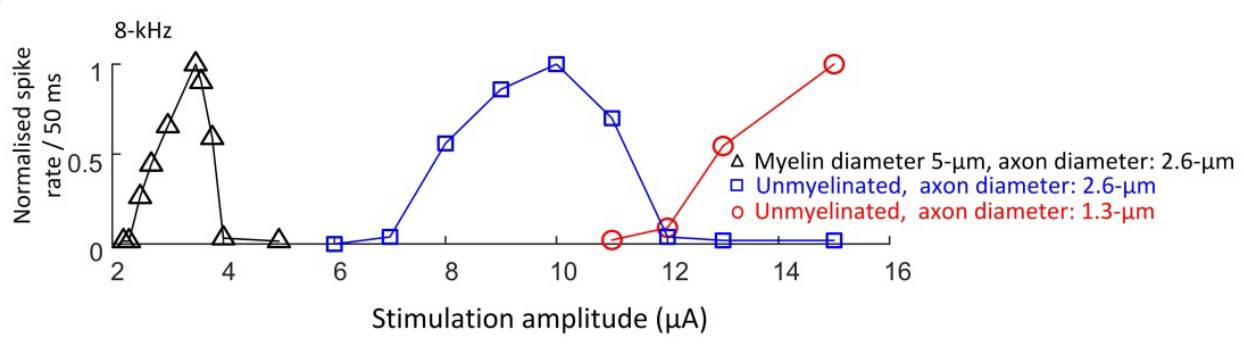

Figure 8: Computational simulation of effects of KES on large and small fibers. (A) Normalized spike rate elicited in the myelinated (A-fiber) and un-myelinated (C-fiber) fiber models using $0.1-\mathrm{KHz}$, $0.5-\mathrm{KHz}, 1-\mathrm{KHz}, 2-\mathrm{KHz}, 4-\mathrm{KHz}, 8-\mathrm{KHz}$ and $12-\mathrm{KHz}$ sinusoid $\mathrm{KES}$, across multiple intensities. (B) Examples of induced AP activity (a) and steady-state activation $\left(\mathrm{m}^{3}\right)$ and inactivation $(\mathrm{h})$ gating variables (b) of sodium current in neurites below the electrode during 8-KHz KES for two types of fibers (A-fiber: panels 1-3, C-fiber: panels 4-6). The time window showing gating variables was indicated by the box in (a1 and a6). The activities were simulated at the stimulation amplitudes indicated by the arrows in panel (A), from low (blue), intermediate (green), to high intensity (yellow). The raw membrane activities with stimulation artifacts were shown using grey shadow. (C) Normalized spike rate elicited in the myelinated A-fiber (black), un-myelinated C-fiber (red), and un-myelinated fiber which has the identical axonal diameter with A fiber (blue). 8-KHz sinusoid KES was used. 


\section{Discussion}

In this study, we manipulated stimulus waveform and pulsing frequency of VNS to attain selective activation of vagal fiber types according to their size, in rats and mice. Selective activation of fibers was assessed over several time scales: by direct recording of fiber-specific compound action potentials elicited by single stimuli, by measurement of vagal fiber-mediated cardiorespiratory responses to short (10-s duration) stimulus trains and by imaging of c-Fos expression in respective neurons in sensory and motor vagal nuclei of the brainstem after 30 minutes of VNS. Selection of waveform or frequency parameters that maximize selectivity for large (A-type), intermediate size (B-type), or small (C-type) fibers was based on calculation of respective selectivity indices, compiled from neural, physiological or c-Fos measurements. Our results in rats and mice indicate that activation of $\mathrm{A}$ - and B-fibers can be selected by waveform manipulation and of C-fibers by frequency manipulation. A-fibers are activated simply by low frequency, low intensity, trains of pulses, and almost independent of selection of waveform (Fig.1C, 2C and 2E/rat, 3C and 3E/mouse). B-fibers are activated by longer square or quasitrapezoidal (QT) pulses (Fig. 1D, 2D and 2F/rat, 3D and 3F/mouse), with optimal stimulus intensity varying between animals. C-fibers are activated by high intensity, high frequency $(>8 \mathrm{KHz}) \mathrm{VNS}$; with those KES parameters, larger size fibers are mostly blocked (Fig. 5E and 5F/rat, 6C and 6D/mouse) whereas small C-fibers are partially activated. Our study shows the fiber selective activation of VNS is feasible in two most commonly-used animal models, and corresponding effects can be quantified and optimized in 3 time scales.

\section{Waveform manipulation for selective activation of $A$ - and B-fibers}

In electrical nerve stimulation using non-penetrating cuff electrodes, fiber recruitment generally follows a size principle: fibers with larger diameters are recruited before, i.e. at lower intensities, compared to fibers with smaller diameters. As a result, even though activation of large fibers is relatively easy, selective targeting of smaller fibers is more challenging. FDA-approved VNS therapies for epilepsy and depression depend on activation of larger fibers, which minimizes the potential for off-target effects ${ }^{54}$. VNS for treatment of heart failure partially relies on activation of intermediate-size B-fibers; targeting of those fibers also produces off-target effects from larger, sensory and motor, vagal fibers with lower threshold, including voice hoarseness, coughing, and neck pain ${ }^{22,55}$. Manipulation of stimulus polarity and waveform has been used to selectively target smaller, slower fibers by supressing conduction in larger, faster fibers. For example, QT pulses delivered in tripolar configuration can effectively supress EMG activity associated with efferent A-fiber recruitment ${ }^{24,28}$. Recently, functional engagement of afferent (fast A-type) or efferent (slow A- or B-type) fibers based on the mechanism of anodal block was demonstrated with appropriate inter-electrode distance and pulse width ${ }^{25}$. Our results are consistent with these findings, as both long duration and QT pulses attain B-fiber activation and its associated HR response, with minimal engagement of A-fibers and evoked EMG, and of Cfibers and associated breathing response (Fig. 2, 3). QT generally outperformed long square pulses (Fig. 2, S3), the main reason being that QT can induce less C-fiber activation under same charge injection, for similar amount of B-fiber activation. One drawback behind waveform manipulation is that different animals had different optimal intensities, especially for B-fibers (Fig. 2, 3, S3). This is possibly related to the proximity between the targeted fiber and the stimulating electrode and is critically dependent on the robustness of the neural interface. As a result, waveform manipulations may be less attractive in therapies relying on long-term implants, which generally show longitudinal changes in stimulation effectiveness ${ }^{56,57}$, or when scaling to 
larger diameter, more complex nerves in large animal models or humans, where there is more anatomical variability in the nerve-electrode interface ${ }^{58}$.

\section{Frequency manipulation for selective activation of C-fibers}

Khz-frequency electrical stimulation (KES) induces reversible inhibition of action potential (AP) propagation in peripheral nerves in a variety of animal models and nerve sizes ${ }^{33}$. However, a possible fiber activation effect resulting from KES is not well understood. Generally, to assess the effect of KES, two methods are most commonly used: measurement of physiological (endorgan) effects, such as muscle force ${ }^{59}$, EMG activity ${ }^{60}$, cardio respiratory function ${ }^{61}$ or cytokine levels ${ }^{62}$, and eCAP nerve responses to single probing stimuli from a secondary stimulation electrode $^{36,62,63}$. Measurement of physiological effects, although necessary, is not sufficient to establish nerve conduction block by KES, nor is nerve block necessarily the cause of a given physiological effect, as this could be achieved through other mechanisms ${ }^{64}$. For example, the Enteromedics vBloc system, used in the treatment of obesity, was thought to induce weight loss by electrically blocking the subdiaphragmatic vagus nerve; recent reports suggest that this effect may be elicited by continuously activating the nerve, as opposed to blocking it ${ }^{35}$. Similarly, eCAPs to probing stimuli assess fiber excitability at the time of delivery of those probing stimuli, and may miss fiber responses during the KES duration. For example, in retinal stimulation, retinal ganglion cell spiking is directly elicited, and assessed during KES, using patch-clamp ${ }^{38}$. In VNS, there is no obvious way to assess in vivo AP conduction in the presence of continuous electrical stimuli, except for patch clamp methods, which are generally not feasible in nerve axons.

In our study, we measured physiological responses and eCAPs to understand fiber recruitment caused by KES. We find that nerve conduction block with KES starts with A-fibers, then happens to B-fibers and finally to C-fibers, consistent with previous studies in rats ${ }^{36,65}$. Slowing in conduction velocity, more prominent in slow fibers, was also observed in our results (Fig. 5B and Fig. S6B), also consistent with previous reports ${ }^{36}$. eCAPs to probing stimuli around KES trains are compatible with sequential conduction block (Fig. 5A-C, S6). However, elicited APs during KES trains cannot be resolved using the eCAP method as activities from multiple fibers are asynchronous ${ }^{66}$, and therefore have to be estimated indirectly, via fiber-mediated physiological responses. Drops in HR, associated primarily with B-fiber activation, are minimal during KES (Fig. 4-6). When they occur, especially at high intensities (Fig. 4D, 6B), they could be explained by activation of afferent $\mathrm{C}$-fibers, leading to a reflexive, less pronounced and slower-onset cardio-inhibition, as opposed to the greater and abrupt-onset HR drop seen with direct B-fiber activation ${ }^{67}$. Robust respiratory responses, mostly associated with C-fiber activation $^{42}$, reach a maximum at an intensity producing only half-maximal block of C-fibers in eCAPs (Fig. 4B-D), raising the possibility of C-fiber action potentials (APs) elicited during KES trains. We also found that, in response to 30 minutes of KES, expression of the immediate early gene c-Fos was stronger in neurons in the NTS compared to neurons in DMV (Fig. 7), a finding compatible with preferential activation of C-fibers by KES.

The intermittent nature of KES trains in our study might be one of the reason that lead to longterm activation rather than purely block, while the latter has been reported in the same animal model using continuous KES stimuli. ${ }^{36,62}$. Through leveraging the duty cycle, or ON/OFF of 
KES, the balance between brief excitation and longer lasting inhibition can be further manipulated and potentially improve the fiber selectivity. The optimal ON-OFF cycle is important as small duty cycle might lead to no selectivity because minimum duration is required to block large fibers, and large duty cycle might end up blocking all large and small fibers.

Therefore, in VNS, and potentially in stimulation of other peripheral nerves, KES delivered in trains is capable of not only providing nerve conduction block, but also fiber-selective activation. An advantage of frequency over waveform manipulations is that optimal KES intensity to achieve preferential C-fiber activation is relatively stable across animals (Fig 5E-F, 6C-D). This feature, along with the possibility for targeting fibers lying in greater distances from the electrode, as is the case with deep brain stimulation ${ }^{68}$ may make frequency manipulation more practical in clinical applications, as it may have a "more forgiving" tolerance with regard to longterm changes in the neural interface and anatomical variations between subjects.

\section{Cellular mechanism of fiber-selectivity of KES}

Previous nerve stimulation studies explored the possibility of selective block in peripheral nerves using KES of $5 \sim 50 \mathrm{kHz}$ frequency ${ }^{69}$ : However, the full range of KES effects, from subthreshold facilitation to suprathreshold block, have not been demonstrated in the vagus, which comprises different fiber types ${ }^{70}$. We used anatomically-accurate, biophysically-plausible computational models of vagal axons to explain the observed selective nerve activation under different KES conditions. In order to isolate the contribution of different stimulation frequencies, our computational model used sinusoid KES to ensure identical total charge across all tested frequencies. Sinusoid and square KES have been used to induce nerve conduction block, and have shown similar blocking mechanisms when the stimulus frequency was high enough ${ }^{71-73}$. Our simulations suggest that myelinated and unmyelinated fibers undergo both activation and block, and that selectivity for one or the other can be attained by modulating stimulus frequency and intensity.

In our model, parameters related to voltage-gated channel kinetics and ion channel distributions were the same for large and small fibers. The simulated fibers differed with regard to their morphology, i.e. axonal size and presence of myelin. Axonal morphology is known to play a role in shaping response properties in different neural systems ${ }^{51-53}$. Our simulations indicate that distinct responses to KES can result from different responses of fibers with different morphologies to the same $\mathrm{KHz}$ stimuli (Fig. 8C). Without considering the influence of myelin, the activation function of an unmyelinated fiber $-\lambda^{2} \cdot\left(\partial^{2} V_{e} / \partial x^{2}\right)$ - will increase with a larger axonal radius, since length constant $\lambda$ is proportional to $\sqrt{\mathrm{r}}$, strengthening the effects of induced depolarization by the electrical stimulation ${ }^{74,75}$. This can also be understood in terms of decreased axial resistance down the nerve fiber with increased radius due to greater fiber crosssectional area: decreased axial resistance leads to greater electrical connection between adjacent segments, preventing the intracellular potential from 'floating' with changes in extracellular potential, rendering the axon more responsive to spatial gradients in the extracellular potential. The effect of myelin does not qualitatively alter but strengthens this effect by significantly increasing the intermodal distance, thereby increasing the voltage gradient on each node ${ }^{74}$. Therefore, the morphological differences between fibers can lead to differentiated thresholds, introducing intensity-dependency of KES. 
The frequency-dependency and fiber-selectivity of KES activation and block could further be explained by the kinetics of ion channels that determine the refractory period and the passive membrane properties of axons that determine the time constant ${ }^{70,76}$. An axon's refractory period, ranging from 1 to $2 \mathrm{~ms}^{77,78}$, imposes a frequency limit of $0.5-1 \mathrm{kHz}$ for stimuli to trigger action potentials (APs) in a one-to-one manner, with one stimulus leading to one $\mathrm{AP}^{70}$. For single stimuli, whether those are pulses or sinusoidal cycles, at intensities below the AP generation threshold for a given fiber type, activation can result from temporal summation of subthreshold membrane voltage fluctuations. When KES stimuli occur faster than the membrane time constant, the resulting charge accumulation together with active mechanisms like suppression of the hyperpolarizing phase, can facilitate spiking ${ }^{79}$ (Fig. 8B-a1 and a6). Instead, at suprathreshold intensities, sodium channel inactivation induced by tonic membrane depolarization is likely the major mechanism underlying action potential block (Fig. 8B-a2 and a3) ${ }^{72,80}$. Intensities that are supra-threshold for large A-fibers block conduction in them (Fig. 8B-a3), while at the same time being sub-threshold for small $\mathrm{C}$-fibers, facilitating asynchronous $\mathrm{C}$-fiber spiking activity (Fig. 8B-a6). By combining the intensity- and frequency-dependencies of KES, the resulting block and activation windows for different fiber types can be separated (Fig. 8A-e to g).

The axonal refractory period might also explain our finding that, during KES, C-fiber eCAPs start to decline at lower intensities than those at which the respective physiological response, i.e. breathing changes, reaches its maximum (yellow bars in Fig. 5C, D). As KES intensity increases, more asynchronous action potentials are elicited from $\mathrm{C}$-fibers, thereby leading to greater changes in breathing. However, that also transiently bring more $\mathrm{C}$-fibers into their absolute refractory period, even for a very short time. That means that fewer C-fibers will be able to generate action potentials in response to the probing stimulus, leading to smaller C-fiber eCAPs.

It is important to note that our simulations are based on single fiber stimulation, while for the in vivo results, eCAPs are obtained from a bundle of mixed fibers. Previously published literature in VN fibers demonstrate that myelinated A-type fibers are evoked in the order A $\alpha$-type, A $\beta$ type, $A \gamma$-type and $A \delta$-type, while our present model only included a generic A-type fiber. In future work, we intend to expand our model to population-based simulation, including additional nerve fiber types and histologically-driven fiber distributions, for more accurate VN fiber modeling. In addition, our model only incorporated basic ionic channel and membrane properties. Newly-reported ionic currents in vagus nerves ${ }^{81}$ along with frequency-dependent membrane capacitance ${ }^{69}$ may also influence the KES-induced selectivity. Additional in silico studies will include nerve-specific ion channel properties to elucidate the mechanisms underlying recorded variances, as well incorporating physiologically relevant experimental designs for further model validation.

\section{Fiber Selective VNS is feasible across animal models}

The recruitment of vagus nerve fibers are well-documented in rodents and have been used to define the classification of different fiber types based on their conduction velocity and anatomical characteristics ${ }^{82}$. In addition, rodent models are ideal to study stimulus protocols specific to each type of $\mathrm{VN}$ fibers, as fascicular structure has been greatly simplified from many (5-10 in human and 20-50 in swine) to few (1-2 in rodent $)^{58}$ at cervical level so each fascicle comprises of all fiber types, while the characteristics of each fiber type remain similar. In our study, we used two commonly-used rodent models, rats and mice, and found that the effects of 
stimulation followed the same trends (Fig. 2-3 and 4; Fig. 5 and 6). This finding likely reflects the fact that waveform and frequency manipulation rely on common neural and biophysical mechanism that are preserved across species. Our simulation data further support that phenomenon of KES selectivity can be simply explained by purely fiber size and myelin structure instead of species-specific ion channels, implying the proposed stimulation strategies might be potentially-translational. However, to translate those findings to the large animal model or human, the identified stimulation strategies need to be retested, as the distance between electrode to the fiber of interest increases and spans a larger range in the complex, multi-fascicle, large diameter nerves ${ }^{83}$. Special designs of electrode, such as multi-contacts ${ }^{84}$, inter-fascicular, or intra-fascicular electrode ${ }^{85}$ might be combined with proposed stimulus and provide further spatial selectivity, resulting in similar fiber selective capability within target fascicle.

\section{Parameters for fiber-selective VNS are similar across rodent species}

Rodent species have been used in the classification of different fiber types based on their conduction velocity and anatomical attributes ${ }^{82}$. In addition, they are used frequently to study fiber engagement by VNS in basic and in translational science studies ${ }^{36,67}$. In our study, we found that the effects of stimulation with waveform and with frequency manipulations followed the same trends in rats and mice (Fig. 2-3 and 4; Fig. 5 and 6). One of the reasons for this is that these manipulations leverage biophysical properties of fibers that are preserved in these 2 species. For example, our simulation data suggest that the phenomenon of KES selectivity can be simply explained by fiber size and the presence of myelin, without the need to invoke speciesspecific ionic conductances. This means that these manipulations may be translatable to the vagus of large animals which, at a microscopic scale, comprises the same fiber types ${ }^{86}$. In addition, the cervical vagus of the mouse and rat is small, $180 \mathrm{um}$ and $260 \mathrm{um}$ in diameter respectively ${ }^{86,87}$, and is organized in one or two fascicles ${ }^{58,86,87}$. As a consequence, all fiber types are represented within a small surface area, lying relatively close to the stimulating electrode, which facilitates a relatively uniform exposure to the generated electric fields in both animal species. However, for these reasons, there will be challenges with translating these manipulations to the human and swine vagus nerve: those nerves have large diameters and complex, multifascicular structures ${ }^{86,87}$, which result in longer distances between the electrodes and the fibers of interest and in intra-neural connective tissue "barriers" to the generated fields. Special electrode designs with multiple contacts ${ }^{84}$, placed epineurally or intraneurally ${ }^{85}$, may have to be combined with the proposed stimulus manipulations to provide additional spatial selectivity with regard to the targeted fascicles.

\section{Fiber Recruitment Calibration with Selectivity Indices}

In our study, we made use of selectivity indices for different fiber types to normalize and compare, across animals, the performance of stimulus manipulations in 3 different time-scales: CAP selectivity index in the millisecond scale (CSI, Fig. 1C-D, 5E), physiological selectivity index in the seconds-to-minute scale (PSI, Fig. 2C-F, 3C-F, 5F) and neural selectivity index in the minutes-to-hour scale (NSI, Fig. 7E). Selectivity indices account for the fact that practically any set of VNS parameters engages multiple fiber populations. For example, it was recently shown that VNS of different stimulus polarities engaged both afferent and efferent fibers, but at different proportions ${ }^{25}$. The CSI and the NSI directly measure the engagement of fibers and the associated neuronal populations, respectively; however, the required measurements are challenging, time-consuming and, in the case of NSI, not feasible in real-time. In contrast, the 
PSI estimates fiber engagement indirectly, by quantifying functional effects of fiber activation on the end organs: neck muscles as a proxy for efferent A-fibers, changes in HR for efferent Bfibers, changes in breathing for afferent C-fibers ${ }^{42}$. Those functional effects can be registered noninvasively in humans and, after additional validation in large animal studies, could be combined into practical bedside systems for calibration and optimization of VNS-based therapies targeting different fibers and organs. For example, the B-fiber PSI could facilitate the optimization of VNS parameters to treat heart failure ${ }^{19}$ or cardiac arrhythmias ${ }^{88}$, whereas the Cfiber PSI could act as an index of therapeutic effect in anti-inflammatory application of VNS ${ }^{18,89}$.

\section{Limitations and Future Work}

Our study has several limitations. First, it was performed under anaesthesia. Isoflurane anesthesia is commonly used in vagus and carotid sinus nerve stimulation studies ${ }^{90}$. Even though is does not alter direct electrical activation of vagus fibers ${ }^{83}$, it has been recently shown that it significantly increases physiological threshold intensities of $\mathrm{VNS}^{57}$. Anesthesia used in our study likely impacts synaptic transmission at the vagal ganglia and blunts baroreflex mediated cardiac responses, even though baroreceptor activation-induced heart rate changes are still observed at high isoflurane concentrations ${ }^{91}$. It is likely that isoflurane suppression of synaptic function will require activation of a larger number of vagal fibers to elicit a similar response compared to the awake state. The use of PT and how it normalizes responses with respect to the applied stimulus intensity makes it physiologically meaningful in anesthetized or awake conditions.

Second, together with selective nerve fiber block and activation, KES transiently evokes neural activity in both afferent and efferent directions ${ }^{33,64}$. In our study, we observed robust laryngeal muscle contraction during KES, especially at intensities above the blocking threshold for A- and B-fibers, an easily-observable indicator for the reversible blocking effect (Fig. S6A). This transient large fiber response, before the complete block, may contribute to the increased c-Fos expression in the ipsilateral DMV. The same phenomenon was observed in the computational model, with transient spikes evoked upon the onset of KES (Fig. 8B, black trace, < 1ms after onset). Though the onset response may lead to side effects in clinical applications, its time course is relatively short and patients might be able to tolerate it. A recently optimization method to eliminate onset response in KES through modulating the intensity at the onset of stimuli might potentially be able to minimize such side effects ${ }^{65}$.

Third, KES may cause nerve damage, as it deposits significantly more power in tissue compared with stimulus in low frequency range. A study in spinal cord stimulation using a phantom and a computational model found that high frequency stimulus induced greater temperature increase, something that might alter the effects of KES over time ${ }^{92}$. In a recent study of vagal KES, it was shown that eCAP responses to probing stimuli during KES were blocked, and eventually recovered up to certain intensity level, beyond which block was irreversible ${ }^{36}$; the higher the intensity, the longer the recovery time ${ }^{36}$. In our study, the duration of KES was shorter (10 s versus $25 \mathrm{~s}$ ) and the interstimulus intervals were longer (5-15 mins versus $100 \mathrm{~s}$ ), something that likely contributed to the full recovery of eCAPs and physiological responses. In our study, all responses elicited below $10 \times \mathrm{PT}$ of $\mathrm{KES}(8-\mathrm{KHz}, 40-\mu \mathrm{s}$ pulse width, $2-2.5 \mathrm{~mA})$ were recoverable, as consistent trend of response remains across trials with fully randomized stimuli (Fig. S5 and S6 respectively). However, at higher intensities, specifically when C-fiber eCAP and the breathing response disappeared nearly completely $(30 \times \mathrm{PT}$ of KES, 6-7.5mA, similarly to Pelot et 
$\mathrm{al}^{36}$ ), both evoked physiological and neural responses became irreversible (data not shown). This finding indicates that complete block of C-fibers in rodents using high intensity KES, while maintaining the integrity of the interface and the nerve, might be challenging.

\section{Author contributions}

YCC conceived and designed experiments, performed experiments, analyzed and interpreted experimental results, and wrote the paper. UA conceived and performed rat experiments. NJ and YCW designed and performed anatomy experiments. IM and AA designed and performed experiments in mice. QL, TG, and SD designed and performed the computational stimulation, and wrote the paper. AG performed rat experiments. AD and JA performed the histological analysis. SD and YAA critically reviewed the paper. SZ conceived and designed experiments, secured funding, analyzed and interpreted experimental results and wrote the paper.

\section{Declaration of interests}

The research was partially supported by a grant to SZ from United Therapeutics Corporation (MD, US). SZ and YCC have a provisional patent application with United Therapeutics Corporation that includes aspects of the research presented in this paper. The other authors declare no conflict of interest.

Data and materials availability: The data that support the findings of this study are available from the corresponding author upon reasonable request. 


\section{References}

1 Johnson, R. L. \& Wilson, C. G. A review of vagus nerve stimulation as a therapeutic intervention. Journal of inflammation research 11, 203-213, doi:10.2147/JIR.S163248 (2018).

2 Pratap, R., Farboud, A., Patel, H. \& Montgomery, P. Vagal nerve stimulator implantation: the otolaryngologist's perspective. European archives of oto-rhino-laryngology: official journal of the European Federation of Oto-Rhino-Laryngological Societies 266, 1455-1459, doi:10.1007/s00405-008-0887-2 (2009).

3 Ben-Menachem, E. Vagus-nerve stimulation for the treatment of epilepsy. The Lancet. Neurology 1, 477-482 (2002).

4 Sackeim, H. A. et al. Vagus nerve stimulation (VNS) for treatment-resistant depression: efficacy, side effects, and predictors of outcome. Neuropsychopharmacology: official publication of the American College of Neuropsychopharmacology 25, 713-728, doi:10.1016/S0893133X(01)00271-8 (2001).

$5 \quad$ Koopman, F. A. et al. Vagus nerve stimulation inhibits cytokine production and attenuates disease severity in rheumatoid arthritis. Proceedings of the National Academy of Sciences of the United States of America 113, 8284-8289, doi:10.1073/pnas.1605635113 (2016).

6 Merrill, C. A. et al. Vagus nerve stimulation in patients with Alzheimer's disease: Additional follow-up results of a pilot study through 1 year. The Journal of clinical psychiatry 67, 1171-1178 (2006).

7 Chakravarthy, K., Chaudhry, H., Williams, K. \& Christo, P. J. Review of the Uses of Vagal Nerve Stimulation in Chronic Pain Management. Current pain and headache reports 19, 54, doi:10.1007/s11916-015-0528-6 (2015).

8 George, M. S. et al. A pilot study of vagus nerve stimulation (VNS) for treatment-resistant anxiety disorders. Brain stimulation 1, 112-121, doi:10.1016/j.brs.2008.02.001 (2008).

9 Tyler, R. et al. Vagus Nerve Stimulation Paired with Tones for the Treatment of Tinnitus: A Prospective Randomized Double-blind Controlled Pilot Study in Humans. Scientific reports 7 , 11960, doi:10.1038/s41598-017-12178-w (2017).

10 De Ferrari, G. M. et al. Long-term vagal stimulation for heart failure: Eighteen month results from the NEural Cardiac TherApy foR Heart Failure (NECTAR-HF) trial. International journal of cardiology 244, 229-234, doi:10.1016/j.ijcard.2017.06.036 (2017).

11 Meyers, E. E., Kronemberger, A., Lira, V., Rahmouni, K. \& Stauss, H. M. Contrasting effects of afferent and efferent vagal nerve stimulation on insulin secretion and blood glucose regulation. Physiological reports 4, doi:10.14814/phy2.12718 (2016).

12 Pardo, J. V. et al. Weight loss during chronic, cervical vagus nerve stimulation in depressed patients with obesity: an observation. International journal of obesity 31, 1756-1759, doi:10.1038/sj.ijo.0803666 (2007).

13 Petkovich, B. W., Vega, J. \& Thomas, S. Vagal modulation of hypertension. Current hypertension reports 17, 532, doi:10.1007/s11906-015-0532-6 (2015).

14 Yoshida, K. et al. Electrical Vagal Nerve Stimulation Ameliorates Pulmonary Vascular Remodeling and Improves Survival in Rats With Severe Pulmonary Arterial Hypertension. JACC. Basic to translational science 3, 657-671, doi:10.1016/j.jacbts.2018.07.007 (2018).

15 Bao, M., Zhou, J. \& Luan, G. M. Treatment of drug-resistant epilepsy with vagus nerve stimulation -- review of 45 cases. Chinese medical journal 124, 4184-4188 (2011). 
16 Yoo, P. B. et al. High-resolution measurement of electrically-evoked vagus nerve activity in the anesthetized dog. Journal of neural engineering 10, 026003, doi:10.1088/17412560/10/2/026003 (2013).

17 Olofsson, P. S. et al. Single-Pulse and Unidirectional Electrical Activation of the Cervical Vagus Nerve Reduces Tumor Necrosis Factor in Endotoxemia. Bioelectronic Medicine 2, 37-42, doi:10.15424/bioelectronmed.2015.00006 (2015).

18 Sundman, E. \& Olofsson, P. S. Neural control of the immune system. Advances in physiology education 38, 135-139, doi:10.1152/advan.00094.2013 (2014).

19 Gold, M. R. et al. Vagus Nerve Stimulation for the Treatment of Heart Failure: The INOVATE-HF Trial. Journal of the American College of Cardiology 68, 149-158, doi:10.1016/j.jacc.2016.03.525 (2016).

20 De Ferrari, G. M. et al. Chronic vagus nerve stimulation: a new and promising therapeutic approach for chronic heart failure. European heart journal 32, 847-855, doi:10.1093/eurheartj/ehq391 (2011).

21 Zannad, F. et al. Chronic vagal stimulation for the treatment of low ejection fraction heart failure: results of the NEural Cardiac TherApy foR Heart Failure (NECTAR-HF) randomized controlled trial. European heart journal 36, 425-433, doi:10.1093/eurheartj/ehu345 (2015).

22 Premchand, R. K. et al. Autonomic regulation therapy via left or right cervical vagus nerve stimulation in patients with chronic heart failure: results of the ANTHEM-HF trial. Journal of cardiac failure 20, 808-816, doi:10.1016/j.cardfail.2014.08.009 (2014).

23 Agostoni, E., Chinnock, J. E., De Daly, M. B. \& Murray, J. G. Functional and histological studies of the vagus nerve and its branches to the heart, lungs and abdominal viscera in the cat. The Journal of physiology 135, 182-205, doi:10.1113/jphysiol.1957.sp005703 (1957).

24 Vuckovic, A., Tosato, M. \& Struijk, J. J. A comparative study of three techniques for diameter selective fiber activation in the vagal nerve: anodal block, depolarizing prepulses and slowly rising pulses. Journal of neural engineering 5, 275-286, doi:10.1088/1741-2560/5/3/002 (2008). Ahmed, U. et al. Anodal block permits directional vagus nerve stimulation. Scientific reports 10, 9221, doi:10.1038/s41598-020-66332-y (2020).

26 Jones, J. F., Wang, Y. \& Jordan, D. Heart rate responses to selective stimulation of cardiac vagal C fibres in anaesthetized cats, rats and rabbits. The Journal of physiology 489 ( Pt 1), 203-214, doi:10.1113/jphysiol.1995.sp021042 (1995).

27 Hennings, K., Arendt-Nielsen, L. \& Andersen, O. K. Orderly activation of human motor neurons using electrical ramp prepulses. Clinical Neurophysiology 116, 597-604, doi:https://doi.org/10.1016/j.clinph.2004.09.011 (2005).

28 Tosato, M., Yoshida, K., Toft, E. \& Struijk, J. J. Quasi-trapezoidal pulses to selectively block the activation of intrinsic laryngeal muscles during vagal nerve stimulation. Journal of neural engineering 4, 205-212, doi:10.1088/1741-2560/4/3/005 (2007).

29 Hennings, K., Arendt-Nielsen, L., Christensen, S. S. \& Andersen, O. K. Selective activation of small-diameter motor fibres using exponentially rising waveforms: A theoretical study. Medical and Biological Engineering and Computing 43, 493-500, doi:10.1007/BF02344731 (2005). Grill, W. M. \& Mortimer, J. T. Stimulus waveforms for selective neural stimulation. IEEE Engineering in Medicine and Biology Magazine 14, 375-385, doi:10.1109/51.395310 (1995).

31 Grill, W. M. \& Mortimer, J. T. Inversion of the current-distance relationship by transient depolarization. IEEE Transactions on Biomedical Engineering 44, 1-9, doi:10.1109/10.553708 (1997).

32 Yoo, P. B. et al. Modulation of heart rate by temporally patterned vagus nerve stimulation in the anesthetized dog. Physiological reports 4, doi:10.14814/phy2.12689 (2016). 
33 Kilgore, K. L. \& Bhadra, N. Reversible nerve conduction block using kilohertz frequency alternating current. Neuromodulation : journal of the International Neuromodulation Society 17, 242-255, doi:10.1111/ner.12100 (2014).

34 Apovian, C. M. et al. Two-Year Outcomes of Vagal Nerve Blocking (vBloc) for the Treatment of Obesity in the ReCharge Trial. Obesity surgery 27, 169-176, doi:10.1007/s11695-016-2325-7 (2017).

35 Johannessen, H. et al. Vagal Blocking for Obesity Control: a Possible Mechanism-Of-Action. Obesity surgery 27, 177-185, doi:10.1007/s11695-016-2278-x (2017).

36 Pelot, N. A. \& Grill, W. M. In vivo quantification of excitation and kilohertz frequency block of the rat vagus nerve. Journal of neural engineering 17, 026005, doi:10.1088/1741-2552/ab6cb6 (2020).

37 Zhao, Y. et al. Neuromuscular Electrical Stimulation with Kilohertz Frequency Alternating Current to Enhance Sensorimotor Cortical Excitability. Conference proceedings : ... Annual International Conference of the IEEE Engineering in Medicine and Biology Society. IEEE Engineering in Medicine and Biology Society. Annual Conference 2018, 2240-2243, doi:10.1109/EMBC.2018.8512855 (2018).

38 Guo, T. et al. Mediating Retinal Ganglion Cell Spike Rates Using High-Frequency Electrical Stimulation. Frontiers in neuroscience 13, doi:10.3389/fnins.2019.00413 (2019).

39 Negi, S., Bhandari, R., Rieth, L. \& Solzbacher, F. In vitro comparison of sputtered iridium oxide and platinum-coated neural implantable microelectrode arrays. Biomedical materials 5, 15007, doi:10.1088/1748-6041/5/1/015007 (2010).

40 Negi, S., Bhandari, R., Rieth, L., Van Wagenen, R. \& Solzbacher, F. Neural electrode degradation from continuous electrical stimulation: comparison of sputtered and activated iridium oxide. Journal of neuroscience methods 186, 8-17, doi:10.1016/j.jneumeth.2009.10.016 (2010).

41 Levy, T. J. et al. An impedance matching algorithm for common-mode interference removal in vagus nerve recordings. Journal of neuroscience methods 330, 108467, doi:10.1016/j.jneumeth.2019.108467 (2019).

42 Chang, Y.C. et al. Quantitative estimation of nerve fiber engagement by vagus nerve stimulation using physiological markers. Brain stimulation 13, 1617-1630, doi:10.1016/j.brs.2020.09.002 (2020).

43 Chang, Y. et al. in 2019 41st Annual International Conference of the IEEE Engineering in Medicine and Biology Society (EMBC). 6278-6281.

44 L Parker, J., H Shariati, N. \& M Karantonis, D. Electrically evoked compound action potential recording in peripheral nerves. Bioelectronics in medicine 1, 71-83, doi:10.2217/bem-2017-0005 (2018).

45 Mclntyre, C. C., Richardson, A. G. \& Grill, W. M. Modeling the excitability of mammalian nerve fibers: influence of afterpotentials on the recovery cycle. Journal of neurophysiology 87, 9951006, doi:10.1152/jn.00353.2001 (2002).

46 Schwarz, J. R., Reid, G. \& Bostock, H. Action-potentials and membrane currents in the human node of ranvier. Eur J Phy 430, 283-292, doi:Doi 10.1007/Bf00374660 (1995).

47 Dokos, S. Modelling Organs, Tissues, Cells and Devices Using MATLAB and COMSOL Multiphysics. (Springer 2017).

48 Lin, Q. et al. A Computational Model of Functionally-distinct Cervical Vagus Nerve Fibers. Annual International Conference of the IEEE Engineering in Medicine and Biology Society. IEEE Engineering in Medicine and Biology Society. Annual International Conference 2020, 2475-2478, doi:10.1109/EMBC44109.2020.9175855 (2020). 
Berthoud, H. R. \& Neuhuber, W. L. Functional and chemical anatomy of the afferent vagal system. Autonomic neuroscience : basic \& clinical 85, 1-17, doi:10.1016/S1566-0702(00)00215-0 (2000).

50 Beaumont, E. et al. Cervical vagus nerve stimulation augments spontaneous discharge in second- and higher-order sensory neurons in the rat nucleus of the solitary tract. American journal of physiology. Heart and circulatory physiology 313, H354-H367, doi:10.1152/ajpheart.00070.2017 (2017).

51 Vetter, P., Roth, A. \& Hausser, M. Propagation of action potentials in dendrites depends on dendritic morphology. J Neurophysio 85, 926-937 (2001).

52 Spruston, N. Pyramidal neurons: dendritic structure and synaptic integration. Nat Rev Neurosci 9 , 206-221, doi:10.1038/nrn2286 (2008).

53 Wang, J., Jacoby, R. \& Wu, S. M. Physiological and morphological characterization of ganglion cells in the salamander retina. Vision Res 119, 60-72, doi:10.1016/j.visres.2015.12.007 (2016). Krahl, S. E., Senanayake, S. S. \& Handforth, A. Destruction of peripheral C-fibers does not alter subsequent vagus nerve stimulation-induced seizure suppression in rats. Epilepsia 42, 586-589, doi:10.1046/j.1528-1157.2001.09700.x (2001).

Banzett, R. B. et al. Cardiorespiratory variables and sensation during stimulation of the left vagus in patients with epilepsy. Epilepsy research 35, 1-11, doi:10.1016/s0920-1211(98)00126-0 (1999). Mughrabi, I. T. et al. An implant for long-term cervical vagus nerve stimulation in mice. bio Rxiv, 2020.2006.2020.160473, doi:10.1101/2020.06.20.160473 (2020).

57 Ahmed, U. et al. Implant- and anesthesia-related factors affecting threshold intensities for vagus nerve stimulation. bioRxiv, 2021.2001.2022.427329, doi:10.1101/2021.01.22.427329 (2021).

58 Settell, M. L. et al. Functional vagotopy in the cervical vagus nerve of the domestic pig: implications for the study of vagus nerve stimulation. Journal of neural engineering 17, 026022 , doi:10.1088/1741-2552/ab7ad4 (2020).

59 Bhadra, N. \& Kilgore, K. L. Direct current electrical conduction block of peripheral nerve. IEEE transactions on neural systems and rehabilitation engineering : a publication of the IEEE Engineering in Medicine and Biology Society 12, 313-324, doi:10.1109/TNSRE.2004.834205 (2004).

60 Patel, Y. A., Kim, B. S., Rountree, W. S. \& Butera, R. J. Kilohertz Electrical Stimulation Nerve Conduction Block: Effects of Electrode Surface Area. IEEE transactions on neural systems and rehabilitation engineering : a publication of the IEEE Engineering in Medicine and Biology Society 25, 1906-1916, doi:10.1109/TNSRE.2017.2684161 (2017).

61 Buckley, U. et al. Bioelectronic neuromodulation of the paravertebral cardiac efferent sympathetic outflow and its effect on ventricular electrical indices. Heart rhythm 14, 1063-1070, doi:10.1016/j.hrthm.2017.02.020 (2017).

62 Patel, Y. A., Saxena, T., Bellamkonda, R. V. \& Butera, R. J. Kilohertz frequency nerve block enhances anti-inflammatory effects of vagus nerve stimulation. Scientific reports 7, 39810, doi:10.1038/srep39810 (2017).

63 Patel, Y. A. \& Butera, R. J. Differential fiber-specific block of nerve conduction in mammalian peripheral nerves using kilohertz electrical stimulation. Journal of neurophysiology 113, 39233929, doi:10.1152/jn.00529.2014 (2015).

64 Patel, Y. A. \& Butera, R. J. Challenges associated with nerve conduction block using kilohertz electrical stimulation. Journal of neural engineering 15, 031002, doi:10.1088/1741-2552/aaadc0 (2018).

65 Yi, G. \& Grill, W. M. Kilohertz waveforms optimized to produce closed-state Na+ channel inactivation eliminate onset response in nerve conduction block. PLoS computational biology 16, e1007766, doi:10.1371/journal.pcbi.1007766 (2020). 
Pena, E., Pelot, N. A. \& Grill, W. M. Non-monotonic kilohertz frequency neural block thresholds arise from amplitude- and frequency-dependent charge imbalance. Scientific reports 11, 5077, doi:10.1038/s41598-021-84503-3 (2021).

67 McAllen, R. M., Shafton, A. D., Bratton, B. O., Trevaks, D. \& Furness, J. B. Calibration of thresholds for functional engagement of vagal $A, B$ and $C$ fiber groups in vivo. Bioelectron Med (Lond) 1, 21-27, doi:10.2217/bem-2017-0001 (2018).

68 Grossman, N. et al. Noninvasive Deep Brain Stimulation via Temporally Interfering Electric Fields. Cell 169, 1029-1041 e1016, doi:10.1016/j.cell.2017.05.024 (2017).

69 Joseph, L. \& Butera, R. J. High-frequency stimulation selectively blocks different types of fibers in frog sciatic nerve. IEEE transactions on neural systems and rehabilitation engineering : a publication of the IEEE Engineering in Medicine and Biology Society 19, 550-557, doi:10.1109/TNSRE.2011.2163082 (2011).

70 Neudorfer, C. et al. Kilohertz-frequency stimulation of the nervous system: A review of underlying mechanisms. Brain stimulation 14, 513-530, doi:10.1016/j.brs.2021.03.008 (2021).

71 Zhang, X., Roppolo, J. R., de Groat, W. C. \& Tai, C. Mechanism of nerve conduction block induced by high-frequency biphasic electrical currents. IEEE transactions on bio-medical engineering $\mathbf{5 3}$, 2445-2454, doi:10.1109/TBME.2006.884640 (2006).

72 Bhadra, N., Lahowetz, E. A., Foldes, S. T. \& Kilgore, K. L. Simulation of high-frequency sinusoidal electrical block of mammalian myelinated axons. Journal of computational neuroscience 22, 313326, doi:10.1007/s10827-006-0015-5 (2007).

73 Tai, C. F., de Groat, W. C. \& Roppolo, J. R. Simulation analysis of conduction block in unmyelinated axons induced by high-frequency biphasic electrical currents. leee Transactions on Biomedical Engineering 52, 1323-1332, doi:10.1109/Tbme.2005.847561 (2005).

74 Rattay, F. Analysis of Models for External Stimulation of Axons. IEEE Transactions on Biomedical Engineering BME-33, 974-977, doi:10.1109/TBME.1986.325670 (1986).

75 Hodgkin, A. L. \& Rushton, W. A. The electrical constants of a crustacean nerve fibre. Proceedings of the Royal Society of Medicine 134, 444-479, doi:10.1098/rspb.1946.0024 (1946). Boulet, J., White, M. \& Bruce, I. C. Temporal Considerations for Stimulating Spiral Ganglion Neurons with Cochlear Implants. Journal of the Association for Research in Otolaryngology: JARO 17, 1-17, doi:10.1007/s10162-015-0545-5 (2016).

77 Tackmann, W. \& Lehmann, H. J. Refractory Period in Human Sensory Nerve Fibres. European neurology 12, 277-292, doi:10.1159/000114626 (1974).

78 Kimura, J., Yamada, T. \& Rodnitzky, R. L. Refractory period of human motor nerve fibres. Journal of neurology, neurosurgery, and psychiatry 41, 784-790, doi:10.1136/jnnp.41.9.784 (1978).

79 Heffer, L. F. et al. Examining the auditory nerve fiber response to high rate cochlear implant stimulation: chronic sensorineural hearing loss and facilitation. Journal of neurophysiology 104, 3124-3135, doi:10.1152/jn.00500.2010 (2010).

80 Kilgore, K. L. \& Bhadra, N. Nerve conduction block utilising high-frequency alternating current. Medical \& biological engineering \& computing 42, 394-406, doi:10.1007/BF02344716 (2004).

81 Pelot, N. A. et al. Excitation properties of computational models of unmyelinated peripheral axons. Journal of neurophysiology 125, 86-104, doi:10.1152/jn.00315.2020 (2021).

82 Woodbury, D. M. \& Woodbury, J. W. Effects of vagal stimulation on experimentally induced seizures in rats. Epilepsia 31 Suppl 2, S7-19, doi:10.1111/j.1528-1157.1990.tb05852.x (1990).

83 Nicolai, E. N. et al. Sources of off-target effects of vagus nerve stimulation using the helical clinical lead in domestic pigs. Journal of neural engineering 17, 046017, doi:10.1088/17412552/ab9db8 (2020). 
84 Aristovich, K. et al. Model-based geometrical optimisation and in vivo validation of a spatially selective multielectrode cuff array for vagus nerve neuromodulation. arXiv:1903.12459 [physics] (2020).

85 Larson, C. E. \& Meng, E. A review for the peripheral nerve interface designer. Journal of neuroscience methods 332, 108523, doi:10.1016/j.jneumeth.2019.108523 (2020).

86 Stakenborg, N. et al. Comparison between the cervical and abdominal vagus nerves in mice, pigs, and humans. Neurogastroenterology \& Motility 32, e13889, doi:https://doi.org/10.1111/nmo.13889 (2020).

87 Pelot, N. A. et al. Quantified Morphology of the Cervical and Subdiaphragmatic Vagus Nerves of Human, Pig, and Rat. Frontiers in neuroscience 14, 601479, doi:10.3389/fnins.2020.601479 (2020).

88 Nasi-Er, B. G. et al. Vagus nerve stimulation reduces ventricular arrhythmias and increases ventricular electrical stability. Pacing and clinical electrophysiology: PACE 42, 247-256, doi:10.1111/pace.13585 (2019).

89 Tanaka, S. et al. Vagus nerve stimulation activates two distinct neuroimmune circuits converging in the spleen to protect mice from kidney injury. Proceedings of the National Academy of Sciences of the United States of America 118, doi:10.1073/pnas.2021758118 (2021).

90 Thompson, G. W. et al. Bradycardia induced by intravascular versus direct stimulation of the vagus nerve. The Annals of thoracic surgery 65, 637-642, doi:10.1016/s0003-4975(97)01351-9 (1998).

91 Bagshaw, R. J. \& Cox, R. H. Baroreceptor control of central and regional hemodynamics with isoflurane in the dog. Acta anaesthesiologica Scandinavica 32, 82-92, doi:10.1111/j.13996576.1988.tb02693.x (1988).

92 Zannou, A. L. et al. Temperature increases by kilohertz frequency spinal cord stimulation. Brain stimulation 12, 62-72, doi:10.1016/j.brs.2018.10.007 (2019). 